Review

\title{
Experimental Investigations of Biological Lubrication at the Nanoscale: The Cases of Synovial Joints and the Oral Cavity
}

\section{Javier Sotres * and Thomas Arnebrant}

Biomedical Sciences, Faculty of Health and Society, Malmö University, Malmö 20506, Sweden; E-Mail: thomas.arnebrant@mah.se

* Author to whom correspondence should be addressed; E-Mail: javier.sotres@mah.se; Tel.: +46-(0)-40-665-7926; Fax: +46-(0)-40-665-8100.

Received: 27 September 2013; in revised form: 24 October 2013 / Accepted: 7 November 2013 / Published: 25 November 2013

\begin{abstract}
Interactions between surfaces are ubiquitous phenomena in living organisms. Nature has developed sophisticated strategies for lubricating these systems, increasing their efficiency and life span. This includes the use of water-based lubricants, such as saliva and synovial fluid. These fluids overcome the limitations of water as a lubricant by the presence of molecules such as proteins, lipids, and polysaccharides. Such molecules may alter surface interactions through different mechanisms. They can increase viscosity enabling fluid-film lubrication. Moreover, molecules adsorb on the surfaces providing mechanisms for boundary lubrication and preventing wear. The mentioned molecules have typical sizes in the nanometer range. Their interaction, as well as the interaction with the entrapping surfaces, takes place through forces in the range of nanonewtons. It is therefore not surprising that the investigation of these systems have been boosted by development of techniques such as scanning probe microscopies and the surface force apparatus which allow studying tribological processes at the nanoscale. Indeed, these approaches have generated an enormous amount of studies over the last years. The aim of this review is to perform a critical analysis of the current stage of this research, with a main focus on studies on synovial joints and the oral cavity.
\end{abstract}

Keywords: biological lubrication; nanotribology; atomic force microscope; surface force apparatus; synovial joint; synovial fluid; oral cavity; saliva 


\begin{abstract}
Abbreviations
AFM: atomic force microscope; BSM: bovine submaxillary mucin; DOPC: 1,2-dioleoyl-sn-glycero-3phosphocholine; DPPC: 1,2-dihexadecanoyl-sn-glycero-3-phosphocholine; EHL: elastohydrodynamic lubrication; GAG: glycosaminoglycan; HA: hyaluronic acid; HSPC: hydrogenated soy phosphatidylcholine; PBS: phosphate buffered saline; POPC: 1-palmitoyl-2-oleoyl-sn-glycero-3phosphocholine; PRP: proline rich protein; SAPL: surface-active phospholipids; SDS: sodium dodecyl sulfate; SFA: surface force apparatus; SUV: single unilamellar vesicle; SZP: superficial zone protein.
\end{abstract}

\title{
1. Introduction
}

Biological surfaces in relative motion are commonly ocurring in nature, which in order to keep friction and wear under control have developed extremely efficient lubrication strategies. In fact, these systems are often lubricated by similar water-based mechanisms [1]. While water itself is a poor lubricant, as its viscosity does not significantly rise under pressure, this has been overcome by nature with the addition of biological molecules such as proteins, lipids, and polysaccharides. This results in very efficient lubricants that allow operation under different regimes. For instance, mucinous glycoproteins which are usually found in these systems can increase the fluid viscosity [2], enabling fluid-film lubrication mechanisms. Additionally, biological molecules adsorb on the interacting surfaces forming films that lubricate in a boundary regime lowering friction and preventing wear [3].

In boundary lubrication the interacting surfaces, or the layers that cover them, establish contact through their asperities. When dealing with biological lubricants, these asperities are mainly the molecules that adsorb on the surface and typically have sizes in the nanometer range. Moreover, these molecules interact between themselves and with the underlying surfaces through forces with values in the nanonewton range. Therefore, in order to unravel the molecular mechanisms that govern biological boundary lubrication it is necessary to employ experimental techniques with the ability to study films of adsorbed biological lubricants at the nanoscale.

In the end of the 20th century, different techniques emerged which allowed the study of surface interactions at the nanoscopic level. Among these techniques, the atomic force microscope (AFM) and the surface force apparatus (SFA) have played an outstanding role in the development of experimental nanobiotribology, mainly because of their ability to study forces and distances at the nanoscale in physiologically relevant conditions. The number of studies where these techniques are applied to study biological lubrication mechanisms grows at a continuously increasing rate every year. To cover all the literature within this field would be an overwhelming task. This review focuses instead on studies dealing with experimental investigations at the nanoscale of two representative parts of the human body which need to be highly lubricated in order to fulfill their physiological functions: synovial joints and the oral cavity. Both of these compartments are lubricated by aqueous lubricants, viz. synovial fluid and saliva respectively. However, they also have marked differences. Synovial joints are closed compartments lined by soft surfaces (cartilages and synovial membranes) while in the oral cavity both soft (buccal mucosa) and hard (teeth) surfaces need to be lubricated. The aim of this review is to summarize the current stage of AFM and SFA research on these systems. 


\section{Some Basic Tribology Concepts}

Even though the basics of tribology are out of the scope of this review, a short overview of its most basic concepts is given in order to facilitate the reading for those non-experts in the field. In general terms, tribology is the science of surfaces in relative motion. This embraces the study of friction, wear and, therefore, lubrication.

Friction may be defined as the resistance offered to the sliding of one surface over another and express itself as a (friction) force opposing the sliding. This resistance may result from different factors such as adhesion, interlocking of asperities and surface deformation. Friction is frequently characterized by the friction coefficient, i.e., the ratio between the force pressing the surfaces together and the force needed to overcome friction. It is important to distinguish between static and kinetic friction. Two opposing surfaces will stick until the sliding force reaches the value of the static friction. Kinetic friction refers to that opposing an on-going relative motion. Typically, the static friction coefficient is larger than the kinetic friction coefficient. In this case, there is a tendency for the motion to be intermittent rather than smooth, a phenomenon known as stick-slip motion. When the initial resistance to motion is overcome the friction force diminishes. This causes an acceleration of the body and, subsequently, the force applied to overcome friction decreases until it is insufficient to overcome the dynamic friction and eventually the relative motion stops. However, kinetic friction exhibits a complicated dependence of the relative velocity and so does the stick-slip phenomenon.

Wear may be defined as the progressive loss of material from surfaces in relative motion. There are many different mechanisms by which wear may occur. Adhesive wear occurs when the adhesion between the interacting bodies is stronger than the cohesive forces of the bodies themselves. Abrasive wear is usually present between surfaces of different hardness. In this case it may occur that the hard surface plow through the softer one. Fatigue wear occurs when cyclic shear gradually weakens the softer surface. Independently of the mechanism, it is friction what eventually causes wear. It has been widely accepted that low friction would prevent wear. However, this is not always the case as it is shown in this review. Low friction does not imply low wear and vice versa.

The term lubrication refers to the process of reducing friction, and/or wear, between relatively moving surfaces by the application of a substance, i.e., the lubricant. Lubrication may take place through different mechanisms/regimes, mainly fluid-film lubrication, boundary lubrication, and the transition between the fluid-film and the boundary regimes, also known as mixed lubrication. Fluid-film lubrication [4] is characterized by the existence of a film of fluid/lubricant that completely separates the interacting surfaces. Therefore, the load on the surfaces is supported by the pressure in the fluid film. This pressure can be maintained by the continuous motion of the surfaces (hydrodynamic lubrication) or by pumping fluid externally (hydrostatic lubrication). Elastohydrodynamic lubrication (EHL) can also be included within this regime and it refers to the situation when, upon large pressures, one or both of the interacting surfaces deform in order to enlarge the load-bearing area. The thickness of the fluid film scales proportionally with the fluid viscosity and the relative velocity, and inversely with the applied load. In fluid-film lubrication, because of the surfaces being completely separated, wear is almost totally prevented, and the sliding resistance is low. At high loads, low sliding speeds and/or low viscosities of the bulk lubricant, the separation between the interacting surfaces decreases until, eventually, they establish contact. In this regime, termed 
boundary lubrication, friction and wear are not governed by the bulk properties of the lubricant but by the properties of thin layers partially formed upon lubricant adsorption. The transition from fluid-film to boundary lubrication, where pressure is borne partially by mechanical contact between the surface asperities and partially by the entrained fluid is termed mixed lubrication. The different lubrication regimes are often visualized in the form of Stribeck curves, where the friction coefficient is plotted in terms of the shear velocity, fluid viscosity, and applied load (Figure 1).

Figure 1. Model Stribeck curve where the friction coefficient ant the fluid film thickness are plotted as a function velocity, fluid viscosity and load for the boundary, lubrication, and hydrodynamic lubrication regimes. Reprinted from [5], with permission from Elsevier.

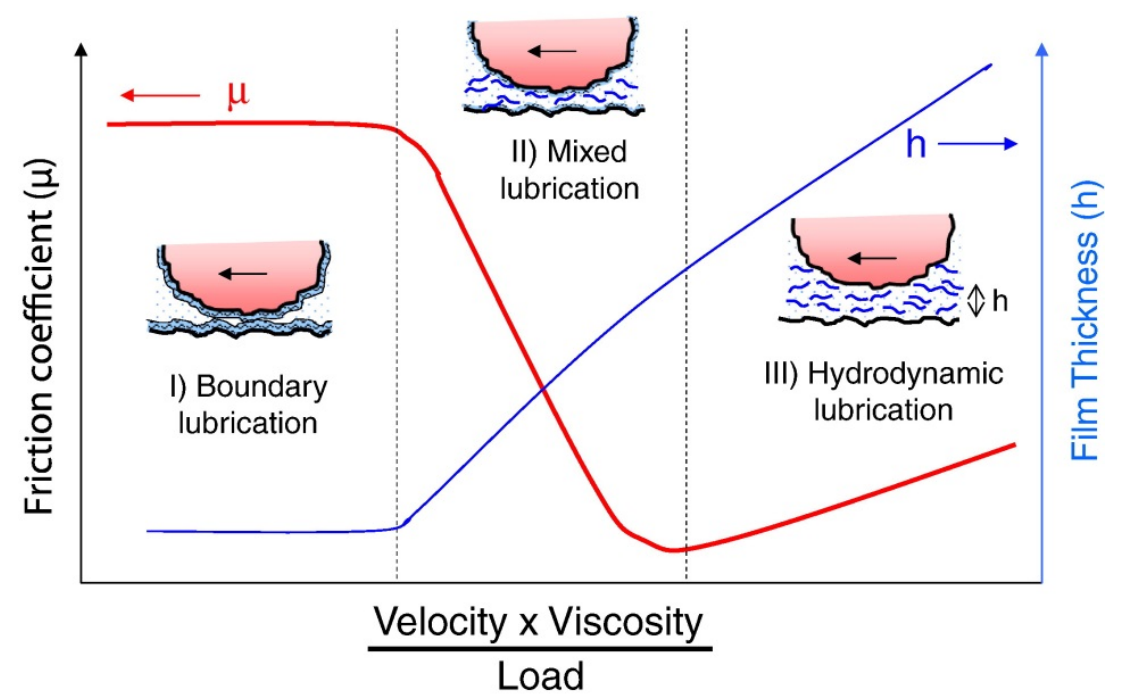

From the previous discussion it is clear that lubrication is highly dependent on the viscoelastic properties of both the bulk lubricant and the adsorbed lubricant films. The fluid-film lubrication regime will prevail for higher loads for lubricants of high viscosity and vice versa. In the boundary lubrication regime the friction force is also highly dependent on the viscoelasticity and plasticity of the adsorbed lubricant films as these properties will determine for instance the contact area between the interacting surfaces or the appearance of plowing effects.

\section{Experimental Techniques for Nanotribological Investigations}

This section presents a short overview of the two experimental techniques most often employed for nanotribological studies, i.e., the atomic force microscope (AFM) and the surface force apparatus (SFA). For a more detailed description, the interested reader is referred to the referenced literature.

\subsection{Atomic Force Microscope (AFM)}

The AFM [6] (Figure 2a) is not a common microscope in the sense that it does not form an image by focusing light or electrons onto a surface as other microscopies do. AFM is based on the measurement of very small forces between the sample surface and a sharp probe/tip (typical apex size is in the range of a few $\mathrm{nm}$ ) mounted on the free end of a flexible cantilever. These forces induce deflections on the cantilever which, through different detection techniques, can be measured with 
sub-angstrom resolution. Standard values for the spring constant of cantilever can be as low as $0.05 \mathrm{~N} \cdot \mathrm{m}^{-1}$, so that forces in the $10-100 \mathrm{pN}$ range can be easily measured. The sample (or the cantilever depending on the specific set-up) is mounted on a piezoelectric tube which can be displaced both in the vertical and lateral dimensions with sub-nanometer resolution. Standard surface imaging is performed by scanning the probe over the sample surface while keeping track of the cantilever deflection or, more often, by keeping the deflection constant by varying the vertical distance between the cantilever and the sample surface. In this way, AFM builds a map of the height of the sample surface. Normal interaction forces between tip and sample can be measured with sub-nanonewton resolution while varying the relative separation between cantilever and sample [7]. This allows for instance the determination of the sample elasticity and of the adhesion force between tip and sample. Torsional deflections of the cantilever can be detected as well [8]. This allows measuring, also with sub-nanonewton resolution, (friction) forces. Tribological studies by means of AFM often include measurements of the dependence of the friction force on the loading (normal) force. However, friction can be highly influenced by the sample topography. In order to eliminate the contribution from the topography "friction loops" are usually acquired, a method that also avoids determining the cantilever torsional deflection corresponding to zero lateral force. Friction loops are acquired by scanning the sample in opposite directions while keeping both the applied load force and the sliding velocity constant. The net friction force is then associated with half the difference between the average lateral forces measured for each of the scanning directions. The nanometer-sized probe can be replaced with a micrometer-sized colloidal particle [9]. In this implementation, from now on referred as colloidal probe spectroscopy, lateral resolution is sacrificed, but in turn the probe geometry is known with high accuracy and, moreover, it is possible to employ probes made of a wide variety of materials.

Figure 2. Schematic drawings of (a) an atomic force microscope (reprinted with permission from [10]); and (b) a surface force apparatus/balance (reprinted by permission from Macmillan Publishers Ltd., Nature [11], copyright 1980).
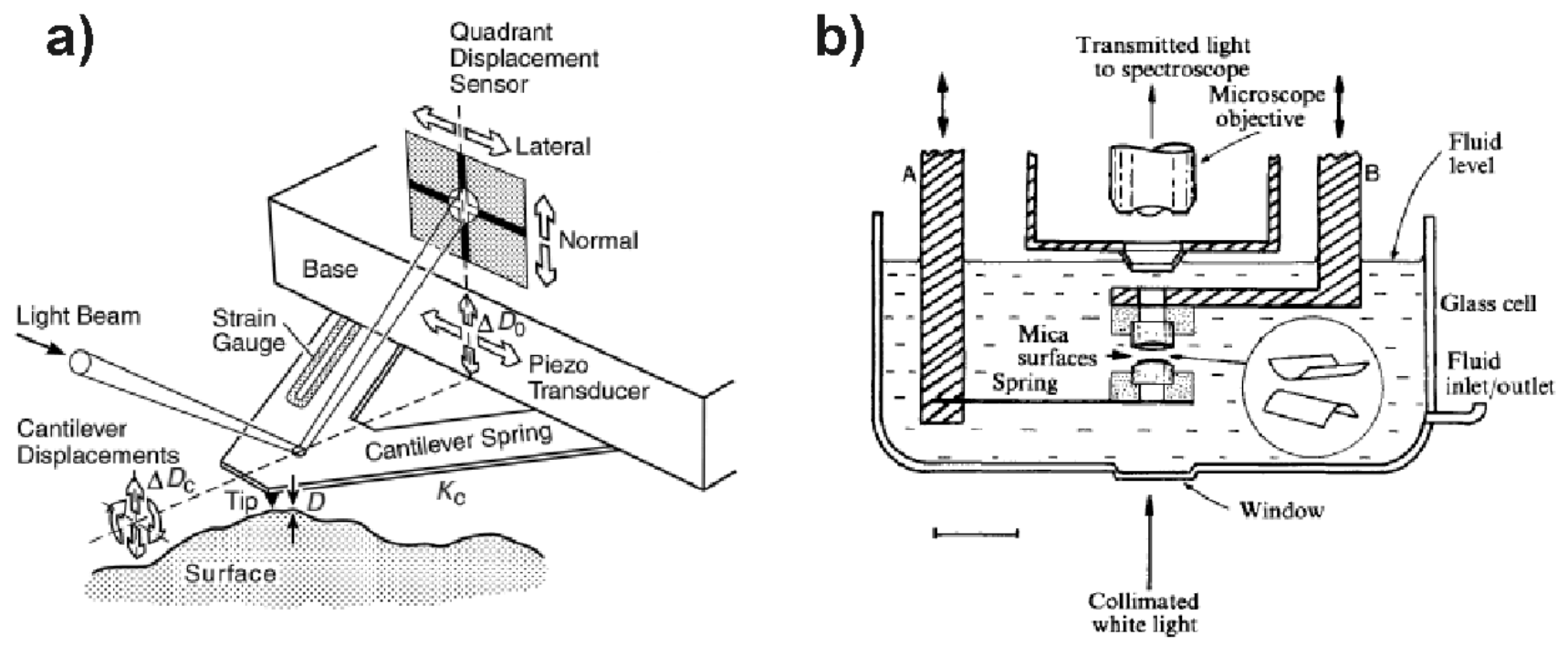


\subsection{Surface Force Apparatus (SFA)}

The surface force apparatus (SFA) [12,13], also known as surface force balance (SFB) depending on the specific set-up characteristics [11] (Figure 2b), measures both the separation and the forces between two curved molecularly smooth surfaces (typical radius of curvature ca. $1 \mathrm{~cm}$ ). These surfaces are typically in a crossed cylinder configuration resulting in a ca. $1 \mathrm{~mm}$ radius circular contact area. The vertical separation between the surfaces is controlled by means of a multiple stage mechanisms which also employs piezoelectric tubes. The surface separation is usually measured by multiple-beam interferometry. This requires transparent surfaces, typically mica. Modified versions of the SFA have been developed in order to employ opaque surfaces [14], replacing the optical distance detection technique by a capacitance method. In both type of systems the separation between the surfaces can be controlled with a precision of ca. $0.1 \mathrm{~nm}$. The sensitivity in normal forces is in the $1-10 \mathrm{nN}$ range. Friction attachments have also been developed for the SFA $[15,16]$, which allow the two surfaces to be sheared laterally while measuring the friction force between them. As in AFM, friction is measured by means of friction loops, i.e., cyclic shear movements in opposite directions at constant applied load and shear velocity, and the net friction force associated with half the difference between the average friction forces measured for each half-cycle. The ranges of friction forces that can be studied are typically in the $10^{2}-10^{8} \mathrm{nN}$ range. The viscosity of a fluid confined between the surfaces can be measured by applying an oscillatory normal motion to one of the surfaces and monitoring the ratio between the induced and resulting oscillation amplitudes [17]. Effective viscous and elastic shear moduli can also be measured by applying an oscillatory shear driving force [18].

\section{3. $A F M$ vs. $S F A$}

Both AFM and SFA can be considered multifunctional tools where the ability to study friction can be combined with the possibility of studying normal interactions, along with topography visualization in the case of the AFM or viscosity and refractive index measurements in the case of the SFA. The ability to measure normal forces is of great help in the understanding of friction data. In both AFM and SFA experiments, friction is measured for a value of an externally applied load force. However, the total normal force acting on the surfaces is the sum of the load force and of the forces resulting from the interaction between the surfaces (which can be determined by both techniques through independent normal force measurements). Therefore, it is important to measure interaction (normal) forces before and after friction measurements in order to correctly interpret friction $v s$. load data. It is important to mention that SFA presents a notorious advantage over AFM when it comes to normal force measurements. In SFA, absolute separations between the interacting surfaces can be measured whereas AFM only allows the determination of relative displacements. This limits AFM not only in determining the thickness of adsorbed layers but also in the interpretation of interaction forces in terms of force laws, especially when the surfaces can be deformed. Concerning interaction forces it is important to mention those arising from the formation of water bridges between the surfaces when working in ambient conditions in a non-zero relative humidity environment $[19,20]$. Such capillary forces exceed all other interaction forces by orders of magnitude, being highly destructive when 
probing biological material. However, capillary forces disappear when working in liquid environment, the most relevant situation in biolubrication studies.

It is also possible to take advantage of the possibility of AFM and SFA to damage samples as this allows studying surface wear. Most commonly wear is inferred from abrupt changes in the friction $v s$. applied load [21], although it can also be characterized from changes in the sample topography in the case of AFM [22,23] or from changes in the shape of the interference fringes in SFA [24].

The AFM and the SFA present some highly marked differences that have to be considered when choosing the appropriate technique to perform an investigation. AFM stands out for its ability to visualize in situ the sample surface and for its high lateral resolution which is in the $\mathrm{nm}$ range as determined by its probe size. On one hand, this allows AFM to perform studies even at the single biomolecule level whereas in SFA interactions are averaged over the whole contact area which is typically in the $\mathrm{mm}^{2}$ range. On the other hand, pressures applied are much higher in AFM than in SFA. As a result of the difference in contact area (ca. 10-100 $\mathrm{nm}^{2}$ in AFM vs. ca. $1 \mathrm{~mm}^{2}$ in SFA), pressures applied in SFA are usually in the MPa range, while in AFM pressures in the GPa range are easily achieved. Friction measured by AFM can be also affected by the local character of this technique. As previously commented, one of the main purposes of friction loops is to eliminate the contribution of the topography to the measured friction. However, the thermal drifts that are commonly present in AFM experiments may prevent an exact spatial matching between trace and retrace data. On the contrary, because of the much larger interacting area, this is less relevant in SFA friction experiments.

\section{Lubrication in Synovial Joints}

\subsection{Physiology and Macrotribology of Synovial Joints}

As early as in the 18th century [25], natural synovial joints were considered as a paradigm of the efficient lubrication strategies developed by nature. Since then, an enormous effort has been devoted to unravel the mechanisms underlying synovial joint lubrication [26-28].

Synovial (or diarthrodial) joints are found at the freely movable articulations of the long bones of the skeleton (hip, knees, shoulders, fingers, etc.). They are known for their extremely effective lubricity combined with incredible load bearing capacity. For instance, the mean pressure on hip joints during the peak of a normal walking cycle is ca. $5 \mathrm{MPa}$ although local pressures of ca. $20 \mathrm{MPa}$ can be achieved [29]. Despite these high pressures, synovial joints exhibit friction coefficients as low as $10^{-3}$ [30-32]. In synovial joints (Figure 3), the ends of opposing bones are covered with a thin layer of cartilage, connected by ligaments and lined by a synovial membrane to create a cavity filled with synovial fluid [33]. Cartilage is a porous material composed of a relatively small number of cells (chondrocytes) embedded in an extracellular matrix. The main components of this matrix/interstitial fluid are water $(60 \%-80 \%$ of the total mass), collagen $(60 \%-70 \%$ of the dry mass), and proteoglycans (mostly aggrecans) (ca. 30\% of the dry mass). The synovial membrane (also known as synovium) lines the inner surface of the joint capsule and covers all intra-articular structures with the exception of the cartilage. Along with the removal of debris from the joint capsule, its main function is the production of synovial fluid. Indeed, the synovial membrane is a membrane permeable up to some extent to plasma, as well as a producer of hyaluronic acid. Effectively, the synovial fluid is essentially a 
dialysate of plasma with the addition of hyaluronic acid (HA), a polysaccharide composed of equimolar quantities of D-glucuronic acid and $N$-acetyl-D-glucosamine residues, although it also contains other components such as a considerable amount of lipids [34,35]. From the rheological aspect, synovial fluid is a non-newtonian fluid with a shear thinning character, i.e., viscosity decreases upon increasing shear rate [36], with viscosities in the $10^{3}-10^{4} \mathrm{mPa} \cdot \mathrm{s}$ range $[32,37,38]$. It is well accepted that this viscous character is imparted by the contained HA molecules [37,39].

Figure 3. A cross-sectional diagram through a synovial joint. Adapted by permission from Macmillan Publishers Ltd., Nature Materials [40], copyright 2013.

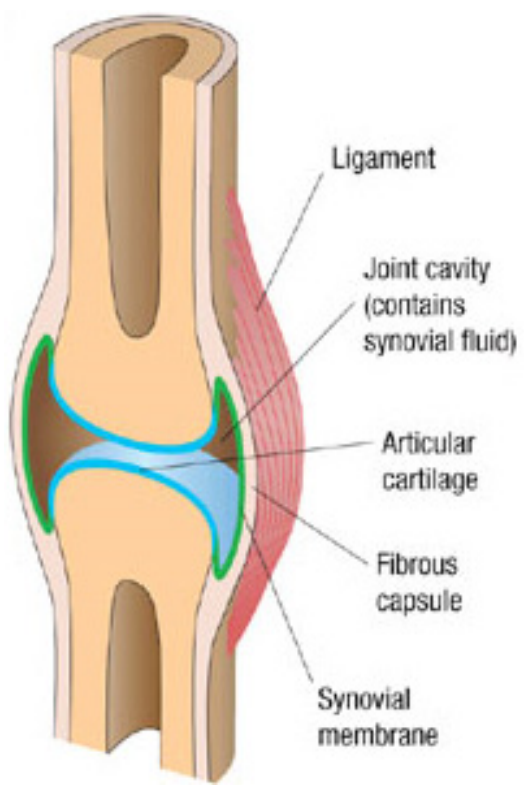

A significantly high number of different lubrication mechanisms have been proposed for synovial joints. These can lay within fluid-film to boundary lubrication, or even a combination of several lubrication mechanisms. Fluid-film lubrication was first thought to govern joint lubrication because of the low friction coefficients observed [37,39]. It has also been proposed that elastohydrodynamic lubrication could play a role as the lubricating pressure could cause a deformation of one or both of the opposing cartilage, thus reducing the velocity needed to maintain a fluid film between the bearing surfaces [41]. However, as with hydrodynamic lubrication, most probably the thickness of the synovial fluid film would not be thick enough to avoid wear of the cartilage asperities. Soon it was recognized that the special properties of cartilage tissue could also play a role in synovial joint lubrication, enabling fluid-film mechanisms. McCutchen [42] proposed a mechanism termed weeping lubrication where the interstitial fluid of hydrated articular cartilage would flow out onto the bearing surface when load is applied. The cartilage would act as a self-pressurizing sponge. When load is released, fluid would flow back into the cartilage.

Eventually [43], hydrodynamic models for joint lubrication were postulated as unlikely as they are not suited to conditions where the motion is reciprocating (as in this situation the relative velocity between the interacting surfaces drops to zero at least twice during each movement cycle). Moreover, hydrodynamic lubrication is not easy to achieve with slow-moving surfaces under heavy-loads. This suggests that boundary lubrication might also play an important role. Boundary lubrication usually 
occurs when the bearing surfaces are separated by a film of molecular dimensions which are somehow attached to them, i.e., a boundary lubricant is needed. This lubricant has to be located at the outer layer of the cartilage surface. Initially, HA was considered the main lubricant component in synovial joints [37]. However, it is, nowadays, generally accepted that HA is involved in fluid-film lubrication as a viscosity modifier but has little boundary lubricating properties [44]. In fact, centrifugation of synovial fluid leads to two separated fractions, one containing mainly HA and the other being mainly a "proteinaceous" fraction [45], the latter shown to be more lubricating [46]. Later on, traditional biological routes of searching for a protein unique to the joint led to the finding of a macromolecular proteoglycan which was given the name of lubricin [47]. Lubricin has been shown to adsorb on the cartilage surface [48]. Indeed, it is also known as "superficial zone protein" (SZP). The major role of lubricin in boundary lubrication is nowadays well-accepted [49]. However, the experimental observation of the hydrophobicity of the articular cartilage [3] contradicts a scheme where a glycosylated and, therefore, hydrophilic protein such as lubricin would be the main boundary lubricant. Additionally, it has also been shown that the "proteinaceous" fraction contains surface-active phospholipids (SAPL) [50]. Interestingly, it was found using enzyme treatment strategies to degrade phospholipids in both the cartilage and in the synovial fluid resulted in a loss of lubrication but that damage to HA had no effect [51]. However, the opposite result has also been reported [52], this leading to some controversy regarding the role of SAPL in joint boundary lubrication [53]. It has been proposed that lubricin could act as a carrier for the SAPL, although other schemes like HA being the carrier for SAPL and this composite adsorbing on lubricin layers at the cartilage surface have also been proposed $[44,54]$. Up to date, a key boundary lubricant has not yet been found. Moreover, it is believed that boundary lubricity may not be attributed to a single component but to the synergistic action of different components of the cartilage outer surface instead.

Fluid-film or boundary lubrication regimes alone may not represent the complex lubrication mechanism in synovial joints. This has led to the appearance of several "mixed" lubrication models [36,55,56]. More recently, the idea of adaptative multi-mode lubrication was proposed [57]. It states that lubrication in synovial joints results from the combination of many different regimes/mechanisms, the nature of which also varies during the joint loading cycle.

\subsection{Nanotribology of Synovial Joints-Cartilage Surfaces}

Due to its feasibility to investigate almost any type of surface, AFM has been used extensively to perform in vitro tribological measurements on dissected cartilage surfaces. In contrast to macroscale measurements, which have been shown to remove not only adsorbed lubricant films but also part of the cartilage itself [58], the high available sample area per probe area ratio in AFM allows to continuously investigate intact zones of the samples.

When investigated on the macroscale, friction on cartilage surfaces exhibit a time-dependent behavior due to the flowing of the interstitial fluid contained in the cartilage tissue away from the contact region. However, in nanoscale friction measurements with the AFM this effect is not observed due to the small contact area and high pressures exerted. As shown by Park and co-workers [59], the friction coefficient measured in AFM experiments corresponds to that measured in equilibrium in macroscale friction measurements. They reported friction coefficients of ca. $10^{-1}$, which are in good 
agreement with those reported by others in AFM investigations of cartilage surfaces [60-62] as well as with those obtained in macroscale measurements after the interstitial fluid pressure drops down, i.e., after the interstitial fluid flows out from the compressed cartilage [63]. Interestingly, Coles and co-workers [62] showed that interfacial shear is the major component of friction measured on cartilage surfaces by means of colloidal probe spectroscopy. They discarded plowing contributions as friction did not show the dependence on sliding velocity expected for this mechanism. Moreover, no correlation was found in this work between friction and cartilage roughness.

The possibility offered by AFM to combine friction measurements with measurement of other quantities such as adhesion, stiffness, and even to visualize, in situ, the sample topography with $\mathrm{nm}$ resolution has been of great aid in unraveling the mechanisms underlying boundary lubrication of cartilage surfaces. Chan and co-workers used this approach along with selective enzymatic digestion of different joint components to investigate the role played by these components in boundary lubrication [60]. Specifically, they digested cartilage lubricin/SZP, HA, and SAPL. The friction coefficient increased after lubricin digestion, but remained unaltered after digestion of HA or SAPL. This supports lubricin being one of the key boundary lubricants. AFM imaging did not demonstrate a consistent trend or significant change of surface roughness for any enzyme treatment. This indicates that changes in friction could not be attributed to modifications of the surface roughness. However, adhesion decreased after lubricin digestion. Therefore, adhesion did not seem to be the dominant friction mechanism either. The authors argued that the observed friction behavior could be due to a dominant plowing mechanism. However, in a later study [61] they also showed that adhesion was lower on those zones of the cartilage which, in in vivo situations, bear higher loads. This was accompanied by lower friction coefficients and a higher concentration of cartilage lubricin as determined by immunohistochemistry techniques. This suggests that the higher content of boundary lubricant at load-bearing cartilage sites preserves synovial joint lubrication, at least up to some extent and, in contrast to their previous findings, by minimizing adhesion.

Recently, friction and wear have also been studied on cartilage surfaces by means of SFA [64]. This was done by gluing a cartilage sample on a glass disc which was then mounted onto the SFA and then tested against another glass disc. In agreement with previous macroscale tribological investigations, at low loads an initially low but temporary increasing friction was observed, this being characteristic of EHL mechanisms maintained by the secretion of interstitial fluid. Eventually this secretion diminishes and the system entered a boundary lubrication regime. They also studied the lubricating role of HA by comparing the tribological behavior of the system before and after HA enzymatic digestion. This affected the system in different ways depending on the load applied. At low loads friction decreased while at high loads it increased. This suggested that in the low load EHL regime HA just play the role of a bulk viscosity modifier, i.e., the decrease in the HA chain length after digestion leads to a lower bulk viscosity, this in turn decreasing friction in the EHL regime. Their results also suggest that under high loads HA becomes anchored to the porous cartilage surface forming a protective layer that, after digestion, could no longer protect the cartilage surface leading to an increase in friction. Indeed, it was possible to monitor surface damage in situ in real time from the friction force traces. When shearing in the transition regime between EHL and boundary lubrication, before HA digestion no sign of damage of the cartilage surface was observed. After digestion, friction in this transition regime decreased but wear on the cartilage surface appeared. This indicated a complex relationship between friction and 
wear but also that the primary role of HA in boundary lubrication is not reducing friction but preventing wear. In a follow-up work [65], friction and wear were studied in a symmetric system, i.e., two cartilage surfaces mounted onto the opposing glass slides of the SFA. In this study, wear on the cartilage surface was characterized after shear by means of interferometry imaging. By means of SFA, they were able to determine that under mild shearing conditions, i.e., not extremely high loads or shearing speeds, stick-slip friction mechanisms can be present, probably originating in interactions and/or entanglements between opposing aggrecan or HA molecules. Moreover, they showed that stick-slip motion led to abrasive wear of the cartilage surface. Indeed, wear showed a higher correlation with the occurrence of stick-slip motion than with the absolute value of friction forces. They also enzymatically digested several joint components (specifically HA, glycosaminoglycans and collagen) to determine their role in stick-slip motion friction and wear prevention. They found that digestion of all studied components lowered the cartilage tribological performance, suggesting that in in vivo situations they work synergistically to prevent wear.

AFM and SFA studies of cartilage surfaces are unavoidably in vitro studies, therefore involving several, and critical, sample preparation steps. Crockett and co-workers used AFM to examine effects of buffer treatment, specifically phosphate buffered saline (PBS), on the structure and mechanical properties of cartilage surface [66]. Initial AFM imaging of cartilage under synovial fluid revealed a gel-like topography. Exchange of synovial fluid by PBS made the surface highly hydrophobic as revealed by AFM adhesion measurements. With time, the cartilage topography changed to a granular one. This was accompanied by a decrease in adhesion indicating that the surface turned from hydrophobic to hydrophilic, and by a decrease in stiffness. This work dramatically showed how critical the handling of the sample is for the reliability of in vitro studies of cartilage surfaces.

\subsection{Nanotribology of Synovial Joints-Reconstituted Model Systems}

The difficulty of studying in vitro cartilage surfaces has favored the investigation of reconstituted systems with the aim of unrevealing the role of the different components of synovial joints in the tribological performance of the whole system.

HA was one of the first molecules whose nanotribological performance was investigated. It was soon realized [67] that, at physiological conditions, HA neither adsorbs nor lubricates the most common surface used in SFA experiments, i.e., mica. Therefore, in order to study friction between HA layers strategies to increase physisorption were employed. This included the ionic binding of the negatively charged HA to (i) to positively charged surfactant bilayers supported on mica substrates, and (ii) to negatively charged mica surfaces via $\mathrm{Ca}^{2+}$ bridges [68]. Normal force measurements showed that these strategies could provide HA layers on the mica surfaces. However, it was also shown that the layers failed in lubricating the surfaces except, perhaps, for very low applied loads. Moreover, it appeared that HA molecules could become forced out from the gap between the surfaces at relatively low pressures ( $>5 \mathrm{~atm}$ ). This led to the conclusion that, in order to hold the HA layers on the sheared surfaces, a must for their lubricating performance, stronger interactions than electrostatics should operate between the molecules and the surfaces. Despite these results, HA could still function as a good boundary lubricant, but for this a stronger force than a mere ionic one would be required to hold the molecules on the surfaces. Therefore, chemisorption strategies were employed. Benz and 
co-workers [69] used two different strategies: (i) Biotynilayed HA was bound a supported lipid bilayer covered with streptavidin; and (ii) HA was directly covalently bound to a lipid bilayer coated mica surface. None of these systems, with friction coefficients of 0.27 and 0.19 , respectively, demonstrated outstanding lubricity. Friction was also measured for hylan (a viscous gel formed by cross-linked HA molecules) covalently attached to the lipid-coated SFA surfaces. However, this system also showed limited lubrication ability with a friction coefficient of 0.15 . In the same study, wear of the chemisorbed layers was characterized by abrupt changes in the friction coefficient. Chemisorbed HA revealed a limited capability to protect the underlying mica surfaces. However, covalently bound hylan showed a much better load-bearing capacity, the surfaces being able to be compressed and sheared at pressures of several hundred atm (10 MPa range) without any damage being observed. Similar results were obtained later on by $\mathrm{Yu}$ and co-workers [70]. They investigated, by means of SFA, the nanotribological performance of chemically grafted and cross-linked HA. In addition to not being extremely lubricating (friction coefficient of ca. 0.5), chemisorbed and cross-linked HA exhibited much higher wear resistance (no surface damage was noticeable up to pressures of $2 \mathrm{MPa}$ ) than physisorbed HA layers. Although it is unlikely that HA at the cartilage surface is chemically cross-linked, it may be networked by other proteins, this being one of the mechanisms used to avoid wear of the cartilage surface as was proposed (vide supra) from SFA experiments on cartilage surfaces [64].

A load-bearing function has also been proposed for lubricin. Jay and co-workers [71] employed AFM to show that lubricin adsorbed on opposing model hydrophobic surfaces and thereby reducing their adhesion. They proposed that the adhesion reduction mechanism was responsible for the role played by lubricin in the reduction in wear observed in macroscale ex vivo measurements between cartilage surfaces. Zappone and co-workers [24] measured normal and friction forces, by means of SFA, between lubricin layers adsorbed on various model planar hydrophilic and hydrophobic surfaces. They also used the interferometric setup of the SFA to characterize wear on the surfaces. They found net repulsive normal force in all cases, supporting the anti-adhesive properties of lubricin layers. They also found that, for loads up to ca. 6 atm (ca. 0.6 MPa), lubricin lubricates hydrophilic surfaces (friction coefficient of 0.02-0.04) better than hydrophobic ones (friction coefficient $>0.3$ ). For higher pressures, wear was observed in the lubricin layers formed on hydrophilic surfaces. However, the layers formed on hydrophobic surfaces did not show wear until 5-15 times higher pressures. These results support again the idea that boundary lubrication and wear protection in articular joints are due to the presence of a biological polyelectrolyte on the cartilage surfaces. The same group performed a follow-up study [72] with the goal of determining the molecular mechanisms underlying the good lubricity of lubricin when coating hydrophilic surfaces. In a simplified picture, lubricin possesses a characteristic molecular structure composed of a long, heavily glycosylated central domain flanked by two nonglycosylated globular end domains. They studied the effect of proteolytic digestion of lubricin by chymotrypsin which separates the globular domains from the glycosylated ones. After in situ digestion, the surface coverage was drastically reduced, the normal forces as measured by means of SFA were altered, and both the coefficient of friction and the wear were dramatically increased. This suggested that, most likely, the enzyme rapidly released the non-adsorbing glycosylated domain into the solution. The anchoring of the glycosylated domain on the surface is thus critical for lubricin to express its full lubricating ability. 
HA-lubricin interaction and its role in synovial joint boundary lubrication have also been investigated. By means of colloidal probe normal force measurements, Chang and co-workers [73] confirmed through the observation of repulsive interactions that lubricin, in contrast to the behavior showed by HA, physisorbed on both model hydrophilic and hydrophobic substrates. Similar measurements on mixtures of lubricin and HA showed repulsive interactions that were only slightly larger than those observed between bare lubricin films, suggesting that the synergistic contributions to the normal force interactions were almost insignificant. In a follow-up study [74], they used colloidal probe spectroscopy to explore the friction behavior of lubricin and HA between model hydrophilic and hydrophobic substrates in physiological buffer solution. Addition of small amounts of lubricin lowered the initially high friction between bare hydrophobic substrates and increased the initially low friction between bare hydrophilic ones. At lubricin concentrations above $200 \mu \mathrm{g} \cdot \mathrm{mL}^{-1}$, the friction behavior between the two types of substrates was similar (friction coefficient of 0.2 ) indicating that this behavior was no longer influenced by the nature of the substrates probably because of being completely covered. In contrast, addition of HA did not alter the frictional behavior between the substrates. Moreover, addition of a physiological mixture of lubricin and HA led to similar friction behavior to that of addition of lubricin alone. This observation suggested that there are also no significant synergistic effect between lubricin and HA on boundary lubrication when only physisorption mechanisms are involved. This is in contrast with what has been found for the interaction between lubricin and chemisorbed HA. Das and co-workers [75] measured normal and friction forces between chemically grafted HA layers on mica surfaces by means of SFA in the presence of lubricin. Addition of lubricin had a pronounced anti-adhesive effect. At low lubricin concentrations, normal forces showed a collapse of the HA layer. This collapse was attributed to the presence of hydrophobic and electrostatic interactions that resulted in bridging of the HA and lubricin molecules. At high lubricin concentrations, the range of the measured repulsive normal forces increased indicating the saturation of the bridging mechanism and the build-up of lubricin on the surface. Increasing the concentration of lubricin decreases the coefficient of friction between the chemisorbed HA layers in a concentration-dependent way (a friction coefficient of 0.37 was reported for a lubricin concentration of $0.5 \mathrm{mg} / \mathrm{L}$, while for $55.5 \mathrm{mg} / \mathrm{mL}$ the friction coefficient decreased to 0.09). Addition of lubricin also increased the threshold load the surfaces could withstand before the inception of damage when they are sheared against each other. Surfaces underwent damage at an applied pressure of about 40 atm, i.e., ca. $4 \mathrm{MPa}$, for $55.5 \mathrm{mg} / \mathrm{L}$ lubricin concentration, which is about twice the pressure the surface could withstand before the onset of damage in the absence of lubricin [70]. The authors could not reproduce any of these results when HA was physisorbed on the mica surfaces. Therefore, a strong surface immobilization of HA is necessary for lubricin to work synergistically with HA and impart efficient lubricating and pressure-bearing properties to the surfaces. This could be the case in the in vivo situation where, under pressure, HA could be trapped in the porous cartilage surface as suggested in the SFA experiments (vide supra) on these systems [64].

The AFM and SFA techniques have also been employed to study the tribological performance of cartilage aggrecan at the nanoscale. Han and co-workers [76] studied friction on a monolayer of chemically end-attached cartilage aggrecan by means of AFM employing both nano-sized tips and micro-sized (colloidal) probes. They observed that friction increased with the ionic strength of the solution (friction coefficient increased from $10^{-2}$ to $10^{-1}$ when increasing the $\mathrm{NaCl}$ concentration from 
$10^{-3}$ to $\left.1 \mathrm{M}\right)$. Moreover, while at low ionic strength friction did not vary with the sliding velocity, a significant dependence with the velocity was found at high ionic strength. These findings were explained in terms of the molecular-level deformation of the aggrecan molecules. The significant dependence of friction with the sliding rate observed at high ionic strengths suggested that time-dependent, e.g., viscoelastic, processes were involved in the deformation of the aggrecan molecules. On the contrary, at low ionic strengths the time-independent (elastic-like) electrostatic forces dominated the interactions between aggrecan molecules. The lubricating performance of reconstructed HA-aggrecan layers, used as a model to emulate the articular cartilage superficial zone structure, has also been studied by means of SFA [77,78]. For this, aggrecan molecules were adsorbed on, and cross-linked via a cartilage link protein to biotinylated HA, which was itself bound on avidin coated mica surfaces. Normal force measurements showed a significant increase in repulsion between the surfaces when aggrecan was added to the HA, indicating a clear interaction between both molecules. They also found the aggrecan-HA complex to be a much better boundary lubricant than the HA alone (friction coefficients of ca. 0.015 and 0.4 respectively). These observations were considered effects attributed largely to the fluid hydration sheath bound to the highly charged glycosaminoglycan (GAG) segments on the aggrecan core protein. However, at pressures of ca. $12 \mathrm{~atm}$ (ca. $1.2 \mathrm{MPa}$ ), a sharp increase in the friction coefficient was observed, indicating surface damage. This suggests that the adsorbed complexes by themselves cannot account for the remarkable boundary lubrication observed in synovial joints up to much higher pressures.

As previously commented, surface active phospholipids are considered strong candidates for being among the lubricating components of synovial joints. In fact, studies on model reconstituted systems have shown phospholipids to be highly lubricating. In this context it is important to consider the fluidity of these systems, solid phases being usually more lubricant than fluid phases $[79,80]$. Friction measured by means of AFM between surfaces with different adsorbed short-chain $\left(\mathrm{C}_{6}-\mathrm{C}_{9}\right)$ phosphatidylcholines revealed friction coefficients in the $10^{-2}-10^{-3}$ range $[81,82]$. Similarly, friction measured in a similar way on 1,2-dioleoyl-sn-glycero-3-phosphocholine (DOPC), which is in the liquid state at room-temperature, layers revealed these interfaces to be also extremely lubricating, with friction coefficients below the resolution limit of the technique [83]. Colloidal probe spectroscopy measurements on DPPC bilayers at high temperature $\left(55{ }^{\circ} \mathrm{C}\right)$, where they are also in a liquid disordered state, provided friction coefficients of ca. $10^{-2}$ [84]. Therefore, from these works it can be inferred that SAPL are probably a key component in synovial joint lubricity. However, free standing SAPL layers possess a main drawback as lubricating systems: They can only stand low pressures before breaking, usually below 1-2 MPa (the work by Liu and co-workers [84] represents a marked exception as they report that DPPC free standing bilayers withstand pressures of ca. $68 \mathrm{MPa}$ ). It has recently been shown that this limitation can be overcome by SAPL vesicles. Close-packed layers of hydrogenated soy phosphatidylcholine (HSPC) single unilamellar vesicles (SUVs) were shown to provide extraordinary good lubricity (friction coefficient in the $10^{-5}-10^{-4}$ range) (Figure 4) up to pressures of at least $12 \mathrm{MPa}[85,86]$. The origin of these low friction coefficients was attributed to a hydration lubrication mechanism [87] afforded by the exposed phosphocholine groups. According to this mechanism the water molecules around these groups would be strongly attached, thus withstanding high normal stresses without being squeezed out, while their rapid relaxation ensures a fluid-like response on shear, thereby leading to very low friction coefficients. As in free standing 
layers, the lubricanting properties of SAPL vesicles were shown to be highly dependent on the lipids phase state. Lower lubricating properties were found for 1-palmitoyl-2-oleoyl-sn-glycero-3phosphocholine (POPC) vesicles [86], a lipid known to be in a liquid-disordered phase at room temperature in contrast to HSPC which is in a solid-ordered phase. In a follow-up work, the same group showed that robustness and lubricity of the SAPL SUVs were, not only determined by the lipid phase, but also by the packing density [88]. In this work they showed that the longer hydrophobic tails, the lower friction coefficients and better load-bearing properties were provided by the SAPL vesicles. The authors discussed that stronger tail-tail hydrophobic interactions resulted in an increase in robustness, this resulting also in more densely packed lipid layers which would therefore be more hydrated, thereby increasing the efficiency of the hydration lubrication mechanism. The lubricant performance of SAPL-HA combined systems has also been studied. In a recent SFA study on DOPC added on chemisorbed and cross-linked HA [70], normal force measurements showed swelling of the HA layers in the presence of the phospholipids, indicating that they adsorbed in the grafted HA layer. However, this adsorption did not result in an improvement of the lubrication properties of the system, yielding a friction coefficient of 0.66. A lower friction coefficient (0.03) was reported in the work of Liu and co-workers for HA adsorbed on DPPC bilayers in a liquid-disordered state [84]. From the reviewed literature, it can be concluded that the role of SAPL on synovial joint lubrication is highly dependent on several factors such as the specific lipids involved (more than 20 lipids have been reported at the cartilage surface [35]), the structure of the lipid systems (e.g., free standing layers $v s$. vesicles), the phase state and packing density of the lipids and their interactions with other synovial joint components.

Figure 4. Friction (shear) vs. load plots obtained by means of SFA for mica surfaces coated by phosphatidylcholine small unilamellar vesicles. Open symbols: first entry to contact point; half-solid symbols: second entry (different symbols refer to different contact points). Reprinted from [85], with permission from Elsevier.

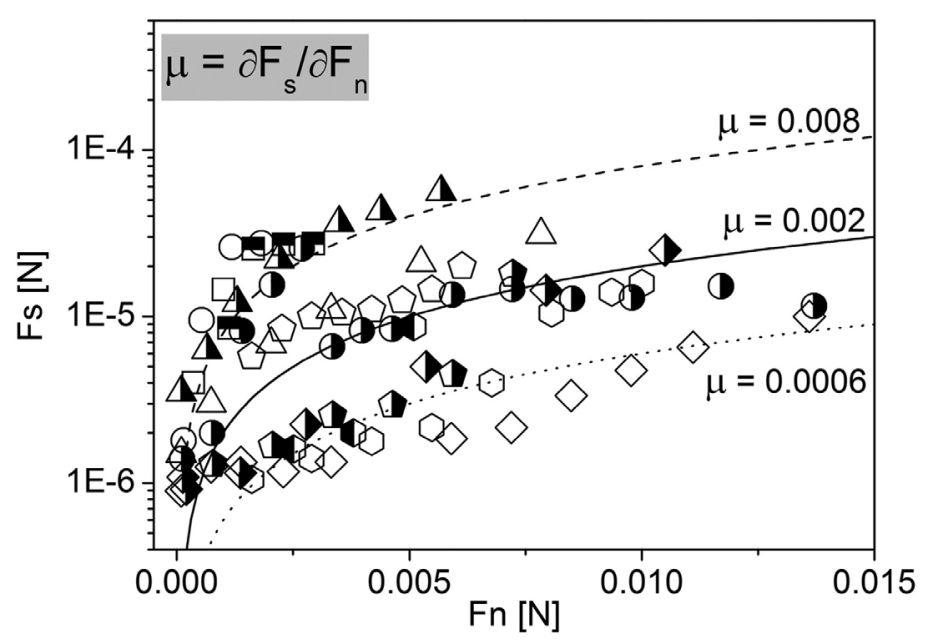




\section{Lubrication in the Oral Cavity}

\subsection{Physiology and Macrotribology of the Oral Cavity}

The oral cavity is the first part of the digestive tube. While its main function is to serve as a food entrance, it also fulfills several other functions. It serves as a secondary respiratory conduit, it enables speech by modifying the sound produced at the vocal folds and it also functions as a chemosensory organ. The oral cavity is bound by the lips, the oropharynx, the cheeks, and the hard and soft palates (Figure 5). Oral surfaces are constantly exposed to mechanical stresses and strains during high-load bearing processes such as mastication [89] as well as during low-load bearing processes such as speech modulation [90]. These processes result in a complex tribological behavior partly because of the high difference in the mechanical properties of the tissues involved. For instance, a stiffness in the 20-85 GPa range [91] has been reported for the main constituent of the outer tooth surface, i.e., enamel, while the stiffness of the softer buccal mucosa was in the 3-20 MPa range [92]. As expected, pressures exerted on the oral cavity are highly dependent on the tissues involved. Pressures of ca. $40 \mathrm{MPa}$ were reported for teeth biting pressures [93,94], whereas for the pressure exerted by the soft tongue surface values in the $10 \mathrm{kPa}-1 \mathrm{MPa}$ range were reported $[95,96]$.

Figure 5. Schematic drawing of the oral cavity [97].

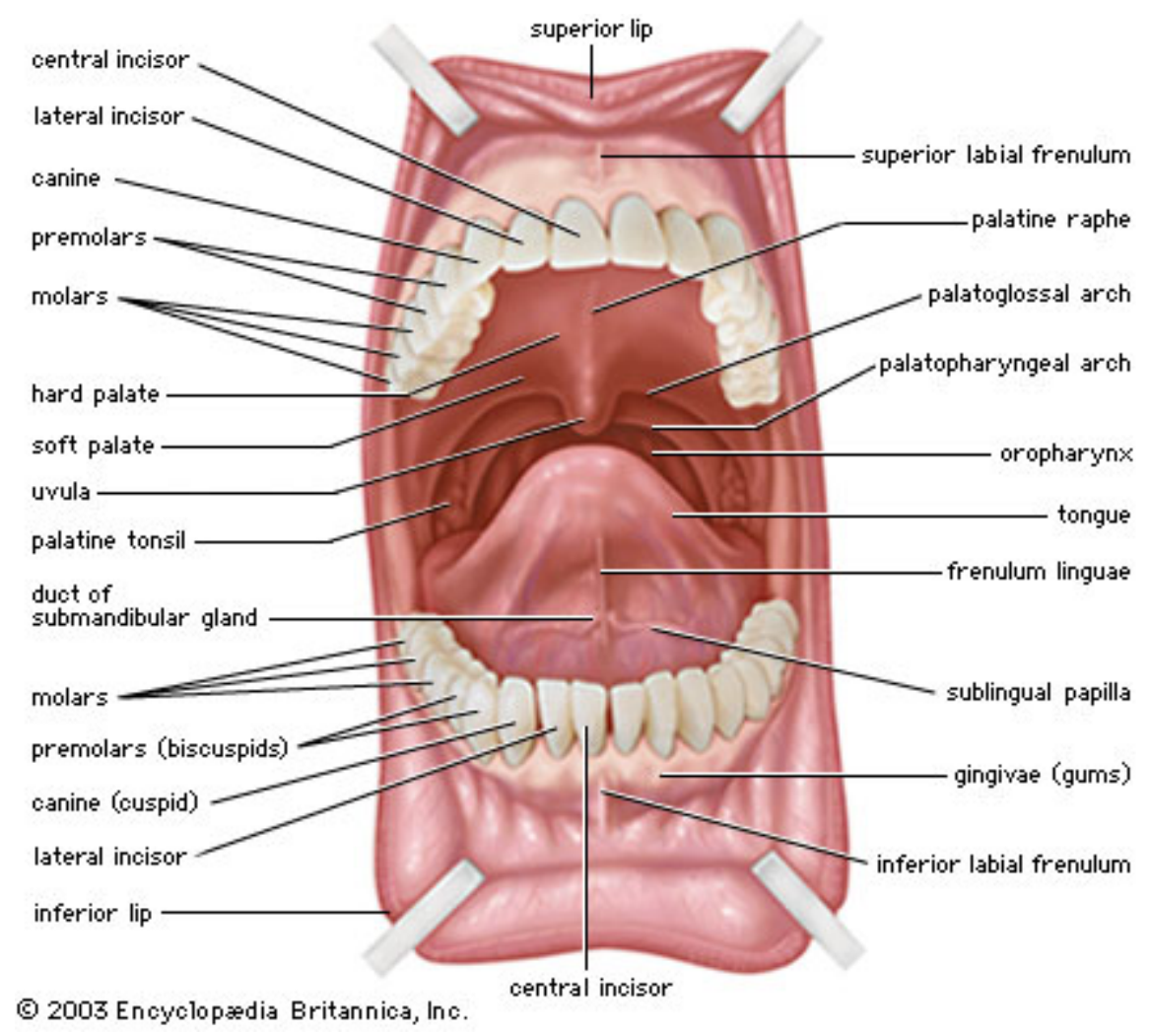

Nature has dealt with lubrication of the oral cavity employing a similar strategy as that developed for lubrication of synovial joints, i.e., by secreting a water-based lubricant into the cavity. The oral lubricant, i.e., saliva, is composed mainly by water (up to 99\%) and proteins, even though it also contains a wide variety of electrolytes and lipids [98]. Similarly to synovial fluid, saliva is a non-newtonian fluid with a shear-thinning character [99]. However, it has a considerable lower 
viscosity, most often reported to be in the 1-10 $\mathrm{mPa} \cdot \mathrm{s}$ range [100-102]. As in the case of synovial fluid, the rheological properties of saliva are strongly influenced by glycans, in this case the high molecular weight mucin MUC5B contributing to its viscosity [103-105] and the smaller mucin MUC7 to its fibrosity [103]. Saliva has been reported to be able to lubricate oral surfaces by operating both in the boundary and in the mixed lubrication regimes [106-109]. Macroscale tribological measurements have provided with a broad range of values for the friction coefficients of surfaces interacting through saliva which, depending on the nature of the surfaces and on the operational parameters, can range from $0.5-0.9$ [109] to $0.02-0.11$ [107]. It is important to note that saliva from different glands appear to have similar lubricating ability [110].

Salivary lubrication is usually attributed to the film of nanometric dimensions that forms immediately upon contact of saliva with all oral surfaces, i.e., the acquired pellicle [111,112]. This was well exemplified by Bongaerts and co-workers [107], who showed that centrifugation and ageing almost completely removed the shear thinning and elastic nature observed for bulk saliva but hardly affected the friction coefficient neither in the boundary nor in the mixed regimes. This indicated that high-molecular weight structures in saliva, which are expected to be responsible for its rheology, are not responsible for its boundary lubricating properties. Moreover, treating the surfaces with SDS (which removes the adsorbed layers) increased the friction coefficient, confirming that lubricity could be mainly attributed to adsorbed proteins.

As in synovial joint research, an extensive search for the components of the pellicle with ability for boundary lubrication has been carried out over the years. This has been proved a difficult task as the composition of the pellicle is far more complex, exhibiting a strong dependence on the physico-chemical properties of the underlying substrate [113]. More than 150 different proteins have been reported as components of the pellicle [114]. Proline rich proteins (PRPs), statherin, and histatins are among the most abundant proteins on enamel surfaces. Mucins, amylase, cystatins, lysozyme, and lactoferrin are also often reported as pellicle components. This compositional complexity may be partly due to the fact the pellicle has to fulfill other functions than lubrication such as reducing the dissolution rate of enamel and modulating the process of mineral precipitation at its surface [115,116].

As in the case of synovial joints, mucins were intuitively though as possible boundary lubricants (even though their interfacial properties are not yet fully understood [117,118]). In fact, they have been proved to provide similar boundary friction coefficients as saliva [106]. However, other pellicle proteins have been shown to provide similar lubricity, as in the case of $\alpha$-amylases [106], or even better, as in the case of statherin or PRPs [119]. Moreover, proteins can act synergistically to enhance the lubrication performance of the adsorbed films as for instance has been shown for the case of PRPs and albumin [120].

However, lubrication does not only refer to decrease friction but also to reduce wear and, as we have seen for synovial joints, these two mechanisms do not always have a direct and clear relationship. While the ability of salivary films to protect the underlying substrates from mechanical wear is well known [121], the mechanical stability of salivary films themselves has only been recently studied. Macakova and co-workers [122] performed macroscale investigations on the lubrication and wear mechanisms of salivary films. They found that the lubrication properties were irreversibly lost when loads in the $10^{-1} \mathrm{MPa}$ were applied, a process they associated with the removal of the adsorbed film. However, they did not observe wear when saliva was present in the bulk, indicating that salivary 
proteins could rapidly re-adsorb from the bulk reservoir and heal wear-induced damage. They also observed that wear was more extensive when exposed to liquids of low ionic strengths, a process they interpreted in terms of a loss of salt ion mediated cross-linking between the adsorbed protein chains.

\subsection{Nanotribology of the Oral Cavity-Whole Saliva Films}

As both the pellicle thickness and the size of its main constituents, i.e., proteins, are in the nm range, the application of both AFM and SFA techniques has boosted research on the molecular mechanisms beyond its tribological performance.

Nylander and co-workers [123] showed, by means of SFA, that salivary films formed on model hydrophilic mica surfaces exhibit non-adhesive repulsive interactions mainly of steric nature, the onset of the repulsion occurring at a substrate-substrate separation of ca. 60-80 nm. A similar behavior was later on observed in a similar SFA study [124], and also in colloidal probe force measurements between salivary films formed on silica [125] and on hydroxyapatite surfaces [126]. Therefore, adhesion could be discarded as the main mechanisms in friction or wear among salivary films.

In contrast with previous macroscale experiments which showed no difference between the lubricant ability of parotid and submandibular-subligual saliva [110], Veeregowda and co-workers [127] showed by means of colloidal probe force measurements that submandibular saliva yielded more lubricious films than parotid saliva, an observation which was attribute to the higher amount of glycosylated mucins in submandibular saliva [128]. In a simultaneous work, the same group studied by means of AFM the effect of different modes of brushing on both the glycosylation and the lubricity of adsorbed salivary films [129]. They found that brushing modes leading to higher degree of deglycosylation, i.e., those removing larger quantities of large glycosylated proteins, result in higher friction coefficients. Therefore, both studies support the role of glycosylated proteins in oral lubrication.

Independently of the underlying mechanism, the lubricating property of saliva has been well proven at the nanoscale. Colloidal probe spectroscopy showed that the presence of salivary films on hydrophilic silica surfaces drops the friction coefficient from ca. 0.66 to ca. 0.03 [125]. The same methodology showed (Figure 6) that, when formed on hydroxyapatite surfaces, salivary films decrease the friction coefficient from ca. 0.44 to ca. 0.2 [126]. In the same study it was observed that SDS treatment of the salivary films increased the friction coefficient to a similar magnitude as in the case of the saliva-free system (Figure 6). This indicated that the adsorbed films removed by the surfactant were the main responsible for saliva lubricity, therefore supporting macroscopic observations (vide supra).

The load bearing properties of salivary films have also been studied at the nanoscale. As previously commented, SFA normal force measurements showed non-adhesive and repulsive steric interactions between adsorbed salivary films. Interestingly, the range of this interaction increases after applying shear [124]. This was consistent with the occurrence of wear based on the ploughing up of material, i.e., shear induces the lifting of material to form debris that extends out from the adsorbed films. However, a threshold pressure value for the occurrence of wear was not provided in this study. 
Figure 6. Friction force $v s$. applied load for two hydroxyapatite microparticles interacting in PBS before and after the formation of salivary films on their surfaces. Friction after SDS treatment is also plotted, indicating that the surfactant-induced removal of the adsorbed layers increased the friction coefficient to a similar magnitude as in the case of the saliva-free system. Reprinted with permission from [126]. Copyright 2008, American Chemical Society.

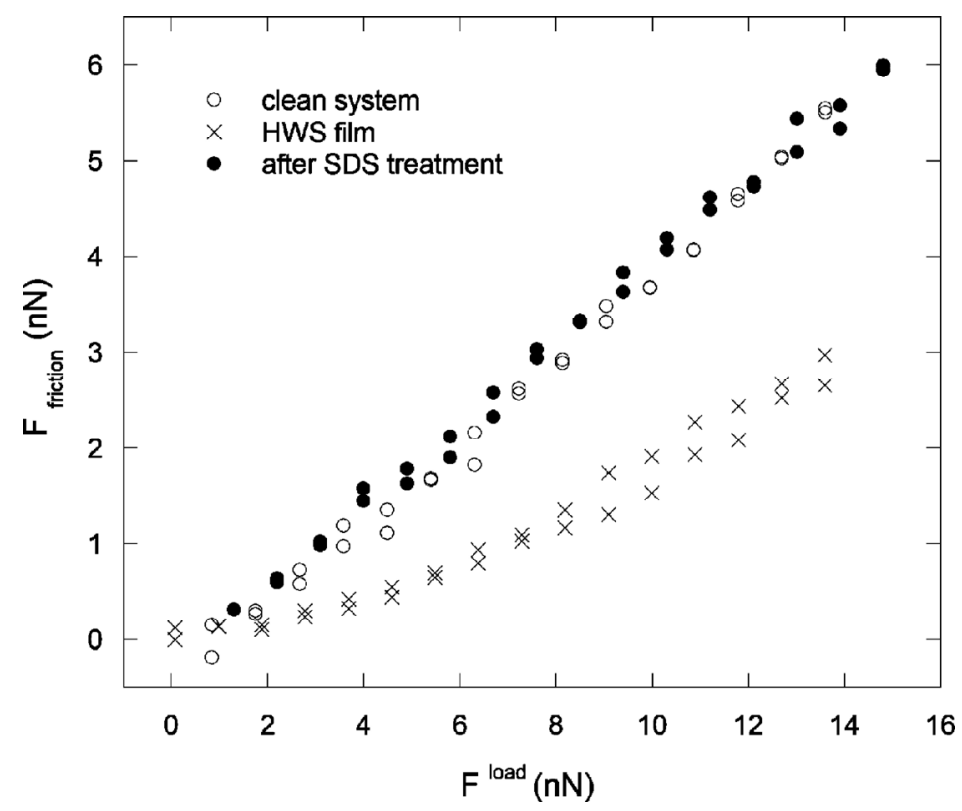

Recently, wear of salivary films has also been characterized by a novel methodology that simultaneously combines the AFM abilities to monitor the sample topography and the friction between tip and sample (Figure 7) [130]. By gradually increasing the applied load, i.e., by scratching the sample, it was possible to detect events such as the rupture of the films as well as their complete removal and, subsequently, the load and friction forces needed for these events to occur. Therefore, in contrast with the more traditional approach, where the onset of wear is characterized by means of the applied normal pressure, this methodology also takes into account the applied shear pressure. This allows, by making use of the von Mises yield criterion, to calculate the yield strength of the scratched films [23]. For salivary films adsorbed on model hydrophobic surfaces in water a yield strength of ca. $120 \mathrm{MPa}$ was found, a value significantly higher that the threshold normal pressure for the onset of pellicle wear reported in macroscale experiments [122]. Interestingly, after the rupture of the films the formation of debris was observed, supporting that wear of salivary films occurs through a ploughing mechanism in agreement with the results from Harvey and co-workers [124]. AFM scratching also provided information on lateral diffusion processes within the pellicle. When, after the complete removal of the films, the load was decreased, the topography images showed the recovery of the surface coverage and friction decreased down to values similar to those measured before the pellicle rupture. Considering that experiments were performed in protein-free solution, this suggested that some of the pellicle components have the ability to diffuse laterally. In a follow-up work it was also shown that the pellicle strength considerably increased with the water wettability, i.e., hydrophilicity, of the underlying substrate [131]. AFM scratching was also employed to determine the mechanisms behind the loss of the pellicle protective ability at low $\mathrm{pH}$ conditions [130]. When rinsed with acidic 
solutions, salivary films formed on hydrophobic substrates became significantly weaker whereas films formed on hydrophilic surfaces collapsed, the homogeneous film being transformed into an incomplete surface coverage of large (height within tens of nanometers) aggregates.

Figure 7. Results from a representative AFM scratching experiment on an adsorbed salivary film. (a) Representative images of the changes in the topography induced by increased applied load during the scratch of a salivary film. Scan area: $2 \mu \mathrm{m} \times 2 \mu \mathrm{m}$. Color scale goes from $0 \mathrm{~nm}$ (black) to $6 \mathrm{~nm}$ (white); (b) Corresponding roughness; and (c) Friction $v s$. load plots. The rupture and removal (sweeping) of the film events are highlighted in both plots. Adapted from [131].

a)

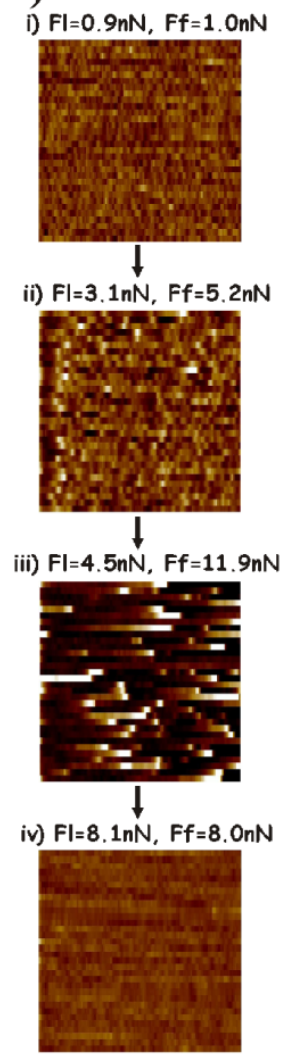

b)

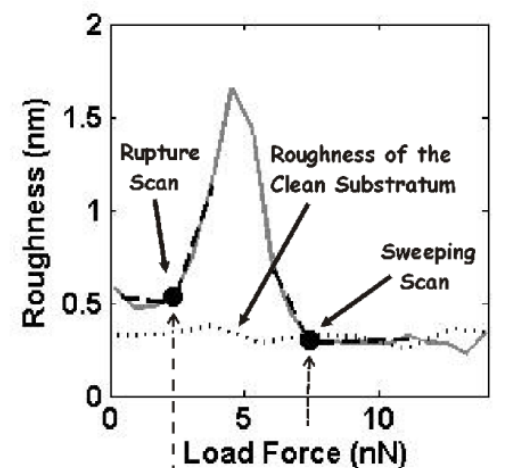

c)

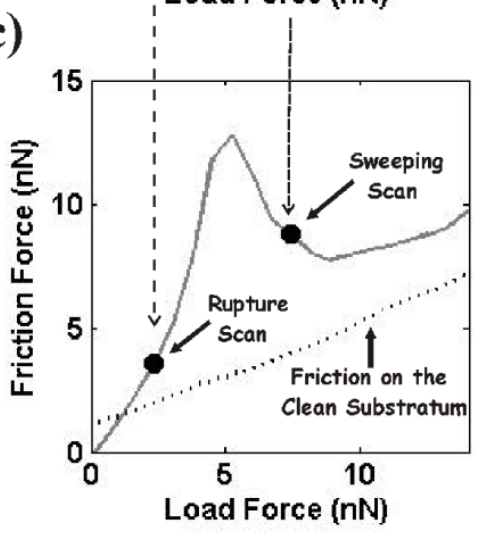

At this point it is fair to mention that tribological studies of pellicles formed in vitro remain controversial as they are known to differ from those formed in vivo [132]. Therefore, as in the case of cartilage surfaces, sample preparation constitutes a critical issue.

\subsection{Nanotribology of the Oral Cavity—Reconstituted Model Systems}

As in synovial joint research, nanoscale techniques have been applied to investigate model systems formed by different components of salivary films in order to unravel their role in oral lubrication. The normal forces between mucins from bovine submaxillary glands (bovine submaxillary mucin, BSM) have been extensively studied by means of both AFM and SFA. These interactions proved to be repulsive and of steric origin. For short adsorption times (30-45 $\mathrm{min}$ ) and mild ionic strength conditions (30 mM monovalent salt) the onset of the repulsion was observed at surface separations in 
the 40-50 nm range [133,134]. However, this onset could be extended to $>100 \mathrm{~nm}$ distances when increasing the adsorption time [133] or decreasing the ionic strength [135,136]. Therefore, mucin films exhibit reasonably similar normal interactions as those shown by salivary films [123]. By means of SFA repulsive steric interactions have also been found for other "lubricant" salivary proteins such as acidic proline rich protein 1 (aPRP-1) [137] and statherin [138]. However, in these cases the onset of the repulsion appeared at significantly shorter distances (ca. $30 \mathrm{~nm}$ for aPRP-1 and ca. $20 \mathrm{~nm}$ for statherin films) than in the case of salivary films. Therefore, mucin appears as the strongest candidate for mediating normal interactions between salivary films.

The lubricant ability of different salivary components has also been probed at the nanoscale. By means of colloidal probe spectroscopy, Hahn-Berg and co-workers [139] investigated lubrication between adsorbed (i) proline-rich protein 1 (PRP-1); (ii) human statherin; and (iii) bovine submaxillary mucin (BSM) layers on silica surfaces. Layers formed by the three different proteins on hydrophilic substrates showed, to various extents, a lubricating effect which was shown to be highly dependent on the surface amount. However, only PRP-1 provided a similar lubricant ability as that found by the same authors for whole saliva [125]. This is in contrast with the findings from Petterson and co-workers [134], who found also by means of colloidal probe spectroscopy that BSM layers adsorbed on similar substrates were highly lubricant with a friction coefficient, i.e., 0.03, similar to that found for salivary films by Hahn-Berg and co-workers [125]. Comparable lubricities for statherin films have not been reported in nanoscale investigations neither in the previously mentioned study nor in a recent work by Harvey and co-workers [138]. By means of SFA they measured friction between statherin layers formed on both model hydrophobic and hydrophilic substrates for which they found friction coefficients of ca. 0.88 and ca. 0.1 respectively. It is interesting to note that this dependence on the substrate wettability was not found in normal force measurements suggesting that aggregates of similar size but with different internal molecular re-arrangements form on both types of substrates.

From the previous studies, it would be reasonable to suggest that mucins are the salivary proteins whose tribological properties most resemble those of salivary films. However, in a yet unpublished study [140] we have investigated wear of BSM films at the nanoscale by means of the AFM scratching methodology [23]. In this study we found that BSM films are significantly weaker than salivary films independently of the substrate employed. Therefore, it is obvious that salivary lubrication, i.e., its ability to reduce friction and wear, results from the synergistic action of several of its components.

\section{Concluding Remarks and Future Perspectives}

The suitability of the AFM and SFA techniques to provide information on the molecular mechanism behind joint and oral lubrication is widely accepted by the scientific community, as inferred from the amount of scientific literature generated in the field. While the key aspects of these types of biological lubrication mechanisms are still not completely clear, it is expected that nanoscale experimental investigations will continue to shed light on them in the near future. It would be daring trying to predict the future development of this field. However, we would like to mention some of the, in our opinion, limitations and challenges faced, as well as possible research directions.

From a technical point of view friction measurements by means of both AFM and SFA are well established. However, the same does not apply to the characterization of wear of boundary lubricant 
films. We have seen that there is usually a discrepancy between the "macroscale" and "nanoscale" threshold pressure/strength values for which the onset of wear is observed. Light should be shed in the origin of these discrepancies. Different methods leading to different results are currently being used to relate the measured forces both in AFM and in SFA to the pressures exerted. This point to the need of developing a general framework for the determination of a pressure-like quantity for characterizing the onset of wear which should include the effect of friction forces/shear.

One of the main limitations of AFM and SFA studies of oral and joint lubrication is that only in vitro investigations are possible. Working in vitro with biological samples such as cartilage surfaces or films adsorbed from whole saliva is not without complications. As commented, the properties of cartilage surfaces are highly dependent on the sample preparation method. In the case of saliva, proteolysis and sedimentation processes are probably among the causes of the known differences between in vivo and in vitro pellicles. The study of reconstituted model systems using biolubricant components, in order to unravel their role in in vivo lubrication, should also be handled with caution. Being able to reconstitute a system which shows good lubricant properties at the nanoscale does not imply that it is present in the in vivo situation. Nevertheless, success in this approach is always beneficial for the development of bio-inspired and bio-compatible lubricating coatings [28].

As commented, lubrication involves minimizing both friction and wear, two quantities that are not necessarily related. Interestingly, from the reviewed literature it is inferred that nature can achieve highly efficient lubrication through different mechanisms in synovial joints compared to in the oral cavity. In the case of joint lubrication, nanoscale investigations have revealed that the glycosylated molecules found in these systems do not themselves account for the very low friction coefficients expected for boundary lubrication in vivo. However, these molecules could rather provide load-bearing properties when trapped by the porous cartilage surface. SAPL are the only joint components that have exhibited in vitro friction coefficients similar to those expected in vivo, a behavior attributed to the hydration lubrication mechanism arising from their highly-hydrated phosphocholine headgroups. Moreover, when forming unilamellar vesicles SAPL also provided excellent load-bearing properties. However, a similar lubricating performance has not yet been observed for SAPL when combined with other synovial joint components.

In contrast to the case of synovial joint lubrication, the long unordered glycosylated molecules found in saliva (mucins) showed similar boundary friction coefficients and normal interactions than films formed upon adsorption of whole saliva. However, recent data showed that mucin films possess much lower load-bearing properties than salivary films, indicating that some other components have to be responsible for this. Interestingly, even though saliva is also known to contain SAPL, their role in oral lubrication has not yet been studied and it would be interesting to explore a possible role of these molecules in oral lubrication. A similar lack of studies is found for the synergistic action of pellicle proteins in salivary lubrication. It is reasonable to anticipate that both these topics will become hot research directions in the near future.

\section{Acknowledgments}

Malmö University and the foundation of Gustaf Theodor Ohlsson are gratefully acknowledged for financial support. 


\section{Conflicts of Interest}

The authors declare no conflict of interest.

\section{References}

1. Lee, S.; Spencer, N.D. Sweet, hairy, soft, and slippery. Science 2008, 319, 575-576.

2. Celli, J.; Gregor, B.; Turner, B.; Afdhal, N.H.; Bansil, R.; Erramilli, S. Viscoelastic properties and dynamics of porcine gastric mucin. Biomacromolecules 2005, 6, 1329-1333.

3. Hills, B.A. Boundary lubrication in vivo. J. Eng. Med. 2000, 214, 83-94.

4. Szeri, A.Z. Hydrodynamic and Elastohydrodynamic Lubrication. In Modern Tribology Handbook; Bhushan, B., Ed.; CRC Press: New York, NY, USA, 2001; pp. 383-453.

5. Coles, J.M.; Chang, D.P.; Zauscher, S. Molecular mechanisms of aqueous boundary lubrication by mucinous glycoproteins. Curr. Opin. Colloid Interface Sci. 2010, 15, 406-416.

6. Binnig, G.; Quate, C.F.; Gerber, C. Atomic force microscope. Phys. Rev. Lett. 1986, 56, 930-933.

7. Butt, H.J.; Cappella, B.; Kappl, M. Force measurements with the atomic force microscope: Technique, interpretation and applications. Surf. Sci. Rep. 2005, 59, 1-152.

8. Mate, C.M.; McClelland, G.M.; Erlandsson, R.; Chiang, S. Atomic-scale friction of a tungsten tip on a graphite surface. Phys. Rev. Lett. 1987, 59, 1942-1945.

9. Ducker, W.A.; Senden, T.J.; Pashley, R.M. Direct measurement of colloidal forces using an atomic force microscope. Nature 1991, 353, 239-241.

10. Leckband, D.; Israelachvili, J. Intermolecular forces in biology. Q. Rev. Biophys. 2001, 34, 105-267.

11. Klein, J. Forces between mica surfaces bearing layers of adsorbed polystyrene in cyclohexane. Nature 1980, 288, 248-250.

12. Israelachvili, J.N.; Adams, G.E. Measurement of forces between two mica surfaces in aqueous electrolyte solutions in the range 0-100 nm. J. Chem. Soc. Faraday Trans. 1 1978, 74, 975-1001.

13. Tabor, D.; Winterton, R.H.S. The direct measurement of normal and retarded van der Waals forces. Proc. R. Soc. Lond. A 1969, 312, 435-450.

14. Tonck, A.; Georges, J.M.; Loubet, J.L. Measurements of intermolecular forces and the rheology of dodecane between alumina surfaces. J. Colloid Interface Sci. 1988, 126, 150-163.

15. Homola, A.M.; Israelachvili, J.N.; Gee, M.L.; McGuiggan, P.M. Measurements of and relation between the adhesion and friction of two surfaces separated by molecularly thin liquid films. J. Tribol. 1989, 111, 675-682.

16. Klein, J.; Kumacheva, E. Simple liquids confined to molecularly thin layers. I. Confinement-induced liquid-to-solid phase transitions. J. Chem. Phys. 1998, 108, 6996-7009.

17. Israelachvili, J.N. Measurement of the viscosity of liquids in very thin films. J. Colloid Interface Sci. 1986, 110, 263-271.

18. Luengo, G.; Schmitt, F.-J.; Hill, R.; Israelachvili, J. Thin film rheology and tribology of confined polymer melts: Contrasts with bulk properties. Macromolecules 1997, 30, 2482-2494. 
19. Colchero, J.; Storch, A.; Luna, M.; Gómez Herrero, J.; Baró, A.M. Observation of liquid neck formation with scanning force microscopy techniques. Langmuir 1998, 14, 2230-2234.

20. Crassous, J.; Charlaix, E.; Loubet, J.L. Capillary condensation between high-energy surfaces. An experimental study with a surface force apparatus. Europhys. Lett. 1994, 28, doi:10.1209/0295-5075/28/1/007.

21. Bhushan, B.; Gupta, B.K.; Azarian, M.H. Nanoindentation, microscratch, friction and wear studies of coatings for contact recording applications. Wear 1995, 181-183, 743-758.

22. Beegan, D.; Chowdhury, S.; Laugier, M.T. Comparison between nanoindentation and scratch test hardness (scratch hardness) values of copper thin films on oxidised silicon substrates. Surf. Coat. Technol. 2007, 201, 5804-5808.

23. Sotres, J.; Barrantes, A.; Arnebrant, T. Friction force spectroscopy as a tool to study the strength and lateral diffusion of protein layers. Langmuir 2011, 27, 9439-9448.

24. Zappone, B.; Ruths, M.; Greene, G.W.; Jay, G.D.; Israelachvili, J.N. Adsorption, lubrication, and wear of lubricin on model surfaces: Polymer brush-like behavior of a glycoprotein. Biophys. J. 2007, 92, 1693-1708.

25. Hunter, W. Of the structure and diseases of articulating cartilages, by William Hunter, surgeon. Phil. Trans. 1742, 42, 514-521.

26. Dėdinaite, A. Biomimetic lubrication. Soft Matter 2012, 8, 273-284.

27. Klein, J. Molecular mechanisms of synovial joint lubrication. J. Eng. Tribol. 2006, 220, 691-710.

28. Neville, A.; Morina, A.; Liskiewicz, T.; Yan, Y. Synovial joint lubrication-Does nature teach more effective engineering lubrication strategies? J. Mech. Eng. Sci. 2007, 221, 1223-1230.

29. Hodge, W.A.; Fijan, R.S.; Carlson, K.L.; Burgess, R.G.; Harris, W.H.; Mann, R.W. Contact pressures in the human hip joint measured in vivo. Proc. Natl. Acad. Sci. USA 1986, 83, 2879-2883.

30. Klein, J. Polymers in living systems: From biological lubrication to tissue engineering and biomedical devices. Polym. Adv. Technol. 2012, 23, 729-735.

31. Linn, F.C. Lubrication of animal joints: II the mechanism. J. Biomech. 1968, 1, 193-205.

32. Wright, V.; Dowson, D. Lubrication and cartilage. J. Anat. 1976, 121, 107-118.

33. Gray, H. Anatomy of the Human Body; Lea \& Febiger: Philadelphia, PA, USA, 1918.

34. Bole, G.G. Synovial fluid lipids in normal individuals and patients with rheumatoid arthritis. Arthritis Rheum. 1962, 5, 589-601.

35. Sarma, A.V.; Powell, G.L.; LaBerge, M. Phospholipid composition of articular cartilage boundary lubricant. J. Orthop. Res. 2001, 19, 671-676.

36. Mcutchen, C.W. Boundary lubrication by synovial fluid: Demonstration and possible osmotic explanation. Fed. Proc. 1966, 25, 1061-1068.

37. Ogston, A.G.; Stanier, J.E. The physiological function of hyaluronic acid in synovial fluid; viscous, elastic and lubricant properties. J. Physiol. 1953, 119, 244-252.

38. Schurz, J.; Ribitsch, V. Rheology of synovial fluid. Biorheology 1987, 24, 385-399.

39. Linn, F.C.; Radin, E.L. Lubrication of animal joints. III. The effect of certain chemical alterations of the cartilage and lubricant. Arthritis Rheum. 1968, 11, 674-682.

40. Setton, L. Polymer therapeutics: Reservoir drugs. Nat. Mater. 2008, 7, 172-174. 
41. Dintenfass, L. Lubrication in synovial joints. Nature 1963, 197, 496-497.

42. Lewis, P.R.; McCutchen, C.W. Experimental evidence for weeping lubrication in mammalian joints. Nature 1959, 184, 1285.

43. Charnley, J. The lubrication of animal joints in relation to surgical reconstruction by arthroplasty. Ann. Rheum. Dis. 1960, 19, 10-19.

44. Crockett, R. Boundary lubrication in natural articular joints. Tribol. Lett. 2009, 35, 77-84.

45. Radin, E.L.; Swann, D.A.; Weisser, P.A. Separation of a hyaluronate-free lubricating fraction from synovial fluid. Nature 1970, 228, 377-378.

46. Swann, D.A.; Radin, E.L. The molecular basis of articular lubrication: I. Purification and properties of a lubricating fraction from bovine synovial fluid. J. Biol. Chem. 1972, 247, 8069-8073.

47. Swann, D.A.; Slayter, H.S.; Silver, F.H. The molecular structure of lubricating glycoprotein-I, the boundary lubricant for articular cartilage. J. Biol. Chem. 1981, 256, 5921-5925.

48. Jones, A.R.C.; Gleghorn, J.P.; Hughes, C.E.; Fitz, L.J.; Zollner, R.; Wainwright, S.D.; Caterson, B.; Morris, E.A.; Bonassar, L.J.; Flannery, C.R. Binding and localization of recombinant lubricin to articular cartilage surfaces. J. Orthop. Res. 2007, 25, 283-292.

49. Jay, G.D.; Torres, J.R.; Warman, M.L.; Laderer, M.C.; Breuer, K.S. The role of lubricin in the mechanical behavior of synovial fluid. Proc. Natl. Acad. Sci. USA 2007, 104, 6194-6199.

50. Schwarz, I.M.; Hills, B.A. Surface-active phospholipid as the lubricating component of lubricin. Br. J. Rheumatol. 1998, 37, 21-26.

51. Hills, B.A.; Monds, M.K. Enzymatic identification of the load-bearing boundary lubricant in the joint. Br. J. Rheumatol. 1998, 37, 137-142.

52. Jay, G.D.; Cha, C.J. The effect of phospholipase digestion upon the boundary lubricating ability of synovial fluid. J. Rheumatol. 1999, 26, 2454-2457.

53. Hills, B.A. Identity of the joint lubricant. J. Rheumatol. 2002, 29, 200-201.

54. Sivan, S.; Schroeder, A.; Verberne, G.; Merkher, Y.; Diminsky, D.; Priev, A.; Maroudas, A.; Halperin, G.; Nitzan, D.; Etsion, I.; et al. Liposomes act as effective biolubricants for friction reduction in human synovial joints. Langmuir 2009, 26, 1107-1116.

55. Hou, J.S.; Mow, V.C.; Lai, W.M.; Holmes, M.H. An analysis of the squeeze-film lubrication mechanism for articular cartilage. J. Biomech. 1992, 25, 247-259.

56. Walker, P.S.; Sikorski, J.; Dowson, D.; Longfield, M.D.; Wright, V. Features of the synovial fluid film in human joint lubrication. Nature 1970, 225, 956-957.

57. Murakami, T.; Higaki, H.; Sawae, Y.; Ohtsuki, N.; Moriyama, S.; Nakanishi, Y. Adaptive multimode lubrication in natural synovial joints and artificial joints. J. Eng. Med. 1998, 212, 23-35.

58. Stachowiak, G.W.; Batchelor, A.W.; Griffiths, L.J. Friction and wear changes in synovial joints. Wear 1994, 171, 135-142.

59. Park, S.; Costa, K.D.; Ateshian, G.A. Microscale frictional response of bovine articular cartilage from atomic force microscopy. J. Biomech. 2004, 37, 1679-1687.

60. Chan, S.M.T.; Neu, C.P.; DuRaine, G.; Komvopoulos, K.; Reddi, A.H. Atomic force microscope investigation of the boundary-lubricant layer in articular cartilage. Osteoarthr. Cartil. 2010, 18, 956-963. 
61. Chan, S.M.T.; Neu, C.P.; Komvopoulos, K.; Reddi, A.H. Dependence of nanoscale friction and adhesion properties of articular cartilage on contact load. J. Biomech. 2011, 44, 1340-1345.

62. Coles, J.M.; Blum, J.J.; Jay, G.D.; Darling, E.M.; Guilak, F.; Zauscher, S. In situ friction measurement on murine cartilage by atomic force microscopy. J. Biomech. 2008, 41, 541-548.

63. Ateshian, G.A. The role of interstitial fluid pressurization in articular cartilage lubrication. J. Biomech. 2009, 42, 1163-1176.

64. Greene, G.W.; Banquy, X.; Lee, D.W.; Lowrey, D.D.; Yu, J.; Israelachvili, J.N. Adaptive mechanically controlled lubrication mechanism found in articular joints. Proc. Natl. Acad. Sci. USA 2011, 108, 5255-5259.

65. Lee, D.W.; Banquy, X.; Israelachvili, J.N. Stick-slip friction and wear of articular joints. Proc. Natl. Acad. Sci. USA 2013, 110, 567-574.

66. Crockett, R.; Roos, S.; Rossbach, P.; Dora, C.; Born, W.; Troxler, H. Imaging of the surface of human and bovine articular cartilage with ESEM and AFM. Tribol. Lett. 2005, 19, 311-317.

67. Tadmor, R.; Chen, N.; Israelachvili, J.N. Thin film rheology and lubricity of hyaluronic acid solutions at a normal physiological concentration. J. Biomed. Mater. Res. 2002, 61, 514-523.

68. Tadmor, R.; Chen, N.; Israelachvili, J. Normal and shear forces between mica and model membrane surfaces with adsorbed hyaluronan. Macromolecules 2003, 36, 9519-9526.

69. Benz, M.; Chen, N.; Israelachvili, J. Lubrication and wear properties of grafted polyelectrolytes, hyaluronan and hylan, measured in the surface forces apparatus. J. Biomed. Mater. Res. A 2004, 71, 6-15.

70. Yu, J.; Banquy, X.; Greene, G.W.; Lowrey, D.D.; Israelachvili, J.N. The boundary lubrication of chemically grafted and cross-linked hyaluronic acid in phosphate buffered saline and lipid solutions measured by the surface forces apparatus. Langmuir 2012, 28, 2244-2250.

71. Jay, G.D.; Torres, J.R.; Rhee, D.K.; Helminen, H.J.; Hytinnen, M.M.; Cha, C.-J.; Elsaid, K.; Kim, K.-S.; Cui, Y.; Warman, M.L. Association between friction and wear in diarthrodial joints lacking lubricin. Arthritis Rheum. 2007, 56, 3662-3669.

72. Zappone, B.; Greene, G.W.; Oroudjev, E.; Jay, G.D.; Israelachvili, J.N. Molecular aspects of boundary lubrication by human lubricin: Effect of disulfide bonds and enzymatic digestion. Langmuir 2007, 24, 1495-1508.

73. Chang, D.P.; Abu-Lail, N.I.; Guilak, F.; Jay, G.D.; Zauscher, S. Conformational mechanics, adsorption, and normal force interactions of lubricin and hyaluronic acid on model surfaces. Langmuir 2008, 24, 1183-1193.

74. Chang, D.P.; Abu-Lail, N.I.; Coles, J.M.; Guilak, F.; Jay, G.D.; Zauscher, S. Friction force microscopy of lubricin and hyaluronic acid between hydrophobic and hydrophilic surfaces. Soft Matter 2009, 5, 3438-3445.

75. Das, S.; Banquy, X.; Zappone, B.; Greene, G.W.; Jay, G.D.; Israelachvili, J.N. Synergistic interactions between grafted hyaluronic acid and lubricin provide enhanced wear protection and lubrication. Biomacromolecules 2013, 14, 1669-1677.

76. Han, L.; Dean, D.; Ortiz, C.; Grodzinsky, A.J. Lateral nanomechanics of cartilage aggrecan macromolecules. Biophys. J. 2007, 92, 1384-1398. 
77. Seror, J.; Merkher, Y.; Kampf, N.; Collinson, L.; Day, A.J.; Maroudas, A.; Klein, J. Articular cartilage proteoglycans as boundary lubricants: Structure and frictional interaction of surface-attached hyaluronan and hyaluronan-aggrecan complexes. Biomacromolecules 2011, 12, $3432-3443$.

78. Seror, J.; Merkher, Y.; Kampf, N.; Collinson, L.; Day, A.J.; Maroudas, A.; Klein, J. Normal and shear interactions between hyaluronan-aggrecan complexes mimicking possible boundary lubricants in articular cartilage in synovial joints. Biomacromolecules 2012, 13, 3823-3832.

79. Corneci, M.-C.; Dekkiche, F.; Trunfio-Sfarghiu, A.M.; Meurisse, M.-H.; Berthier, Y.; Rieu, J.P. Tribological properties of fluid phase phospholipid bilayers. Tribol. Int. 2011, 44, 1959-1968.

80. Trunfio-Sfarghiu, A.-M.; Berthier, Y.; Meurisse, M.-H.; Rieu, J.-P. Role of nanomechanical properties in the tribological performance of phospholipid biomimetic surfaces. Langmuir 2008, 24, 8765-8771.

81. Ducker, W.A.; Clarke, D.R. Controlled modification of silicon nitride interactions in water via zwitterionic surfactant adsorption. Colloids Surf. A 1994, 93, 275-292.

82. Ducker, W.A.; Luther, E.P.; Clarke, D.R.; Lange, F.F. Effect of zwitterionic surfactants on interparticle forces, rheology, and particle packing of silicon nitride slurries. J. Am. Ceram. Soc. 1997, 80, 575-583.

83. Grant, L.M.; Tiberg, F. Normal and lateral forces between lipid covered solids in solution: Correlation with layer packing and structure. Biophys. J. 2002, 82, 1373-1385.

84. Liu, C.; Wang, M.; An, J.; Thormann, E.; Dedinaite, A. Hyaluronan and phospholipids in boundary lubrication. Soft Matter 2012, 8, 10241-10244.

85. Goldberg, R.; Schroeder, A.; Barenholz, Y.; Klein, J. Interactions between adsorbed hydrogenated soy phosphatidylcholine (HSPC) vesicles at physiologically high pressures and salt concentrations. Biophys. J. 2011, 100, 2403-2411.

86. Goldberg, R.; Schroeder, A.; Silbert, G.; Turjeman, K.; Barenholz, Y.; Klein, J. Boundary lubricants with exceptionally low friction coefficients based on 2D close-packed phosphatidylcholine liposomes. Adv. Mater. 2011, 23, 3517-3521.

87. Gaisinskaya, A.; Ma, L.; Silbert, G.; Sorkin, R.; Tairy, O.; Goldberg, R.; Kampf, N.; Klein, J. Hydration lubrication: Exploring a new paradigm. Faraday Discuss. 2012, 156, 217-233.

88. Sorkin, R.; Kampf, N.; Dror, Y.; Shimoni, E.; Klein, J. Origins of extreme boundary lubrication by phosphatidylcholine liposomes. Biomaterials 2013, 34, 5465-5475.

89. Dejak, B.; Młotkowski, A.; Romanowicz, M. Finite element analysis of stresses in molars during clenching and mastication. J. Prosthet. Dent. 2003, 90, 591-597.

90. McGlone, R.E.; Proffit, W.R. Correlation between functional lingual pressure and oral cavity size. Cleft Palate J. 1972, 9, 229-235.

91. Zhou, Z.R.; Zheng, J. Tribology of dental materials: A review. J. Phys. D: Appl. Phys. 2008, 41, doi:10.1088/0022-3727/41/11/113001.

92. Goktas, S.; Dmytryk, J.J.; McFetridge, P.S. Biomechanical behavior of oral soft tissues. J. Periodontol. 2011, 82, 1178-1186.

93. Alkan, A.; Keskiner, I.; Arici, S.; Sato, S. The effect of periodontal surgery on bite force, occlusal contact area and bite pressure. J. Am. Dent. Assoc. 2006, 137, 978-983. 
94. Hidaka, O.; Iwasaki, M.; Saito, M.; Morimoto, T. Influence of clenching intensity on bite force balance, occlusal contact area, and average bite pressure. J. Dent. Res. 1999, 78, 1336-1344.

95. Kieser, J.; Singh, B.; Swain, M.; Ichim, I.; Waddell, J.N.; Kennedy, D.; Foster, K.; Livingstone, V. Measuring intraoral pressure: Adaptation of a dental appliance allows measurement during function. Dysphagia 2008, 23, 237-243.

96. Yoshikawa, M.; Yoshida, M.; Tsuga, K.; Akagawa, Y.; Groher, M. Comparison of three types of tongue pressure measurement devices. Dysphagia 2011, 26, 232-237.

97. Tongue. Encyclopædia Britannica Online. Available online: http://www.Britannica.Com/ ebchecked/media/68879/anterior-view-of-the-oral-cavity (accessed on 11 July 2013).

98. Humphrey, S.P.; Williamson, R.T. A review of saliva: Normal composition, flow, and function. J. Prosthet. Dent. 2001, 85, 162-169.

99. Schwarz, W.H. The rheology of saliva. J. Dent. Res. 1987, 66, 660-666.

100. Christersson, C.E.; Lindh, L.; Arnebrant, T. Film-forming properties and viscosities of saliva substitutes and human whole saliva. Eur. J. Oral Sci. 2000, 108, 418-425.

101. Haward, S.; Odell, J.; Berry, M.; Hall, T. Extensional rheology of human saliva. Rheol. Acta 2011, 50, 869-879.

102. Rantonen, P.J.; Meurman, J.H. Viscosity of whole saliva. Acta Odontol. Scand. 1998, 56, 210-214.

103. Inoue, H.; Ono, K.; Masuda, W.; Inagaki, T.; Yokota, M.; Inenaga, K. Rheological properties of human saliva and salivary mucins. J. Oral Biosci. 2008, 50, 134-141.

104. Wickström, C.; Christersson, C.; Davies, J.R.; Carlstedt, I. Macromolecular organization of saliva: Identification of "insoluble" MUC5B assemblies and non-mucin proteins in the gel phase. Biochem. J. 2000, 351, 421-428.

105. Wickström, C.; Davies, J.R.; Eriksen, G.V.; Veerman, E.C.; Carlstedt, I. MUC5B is a major gel-forming, oligomeric mucin from human salivary gland, respiratory tract and endocervix: Identification of glycoforms and C-terminal cleavage. Biochem. J. 1998, 334, 685-693.

106. Aguirre, A.; Mendoza, B.; Reddy, M.S.; Scannapieco, F.A.; Levine, M.J.; Hatton, M.N. Lubrication of selected salivary molecules and artificial salivas. Dysphagia 1989, 4, 95-100.

107. Bongaerts, J.H.H.; Rossetti, D.; Stokes, J.R. The lubricating properties of human whole saliva. Tribol. Lett. 2007, 27, 277-287.

108. Prinz, J.F.; de Wijk, R.A.; Huntjens, L. Load dependency of the coefficient of friction of oral mucosa. Food Hydrocoll. 2007, 21, 402-408.

109. Reeh, E.S.; Aguirre, A.; Sakaguchi, R.L.; Rudney, J.D.; Levine, M.J.; Douglas, W.H. Hard tissue lubrication by salivary fluids. Clin. Mater. 1990, 6, 151-161.

110. Aguirre, A.; Mendoza, B.; Levine, M.J.; Hatton, M.N.; Douglas, W.H. In vitro characterization of human salivary lubrication. Arch. Oral Biol. 1989, 34, 675-677.

111. Dawes, C.; Jenkins, G.N.; Tonge, C.H. The nomenclature of the integuments of the enamel surface of teeth. Br. Dent. J. 1963, 115, 65-68.

112. Vassilakos, N.; Arnebrant, T.; Glantz, P.O. Adsorption of whole saliva onto hydrophilic and hydrophobic solid surfaces: Influence of concentration, ionic stength and $\mathrm{pH}$. Scand. J. Dent. Res. 1992, 100, 346-353.

113. Svendsen, I.E.; Lindh, L. The composition of enamel salivary films is different from the ones formed on dental materials. Biofouling 2009, 25, 255-261. 
114. Siqueira, W.L.; Custodio, W.; McDonald, E.E. New insights into the composition and functions of the acquired enamel pellicle. J. Dent. Res. 2012, 91, 1110-1118.

115. Hannig, M.; Joiner, A. The structure, function and properties of the acquired pellicle. Monogr. Oral Sci. 2006, 19, 29-64.

116. Lendenmann, U.; Grogan, J.; Oppenheim, F.G. Saliva and dental pellicle-A review. Adv. Dent. Res. 2000, 14, 22-28.

117. Dédinaité, A. Interfacial Properties of Mucins. In Encyclopedia of Surface and Colloid Science, 2nd ed.; Taylor \& Francis: New York, NY, USA, 2011; pp. 1-17.

118. Svensson, O.; Arnebrant, T. Mucin layers and multilayers-Physicochemical properties and applications. Curr. Opin. Colloid Interface Sci. 2010, 15, 395-405.

119. Douglas, W.H.; Reeh, E.S.; Ramasubbu, N.; Raj, P.A.; Bhandary, K.K.; Levine, M.J. Statherin: A major boundary lubricant of human saliva. Biochem. Biophys. Res. Commun. 1991, 180, 91-97.

120. Hatton, M.N.; Loomis, R.E.; Levine, M.J.; Tabak, L.A. Masticatory lubrication. The role of carbohydrate in the lubricating property of a salivary glycoprotein-albumin complex. Biochem. J. 1985, 230, 817-820.

121. Joiner, A.; Schwarz, A.; Philpotts, C.J.; Cox, T.F.; Huber, K.; Hannig, M. The protective nature of pellicle towards toothpaste abrasion on enamel and dentine. J. Dent. 2008, 36, 360-368.

122. Macakova, L.; Yakubov, G.E.; Plunkett, M.A.; Stokes, J.R. Influence of ionic strength on the tribological properties of pre-adsorbed salivary films. Tribol. Int. 2011, 44, 956-962.

123. Nylander, T.; Arnebrant, T.; Glantz, P.O. Interactions between salivary films adsorbed on mica surfaces. Colloids Surf. A 1997, 129-130, 339-344.

124. Harvey, N.M.; Yakubov, G.E.; Stokes, J.R.; Klein, J. Lubrication and load-bearing properties of human salivary pellicles adsorbed ex vivo on molecularly smooth substrata. Biofouling 2012, 28, 843-856.

125. Hahn Berg, I.C.; Rutland, M.W.; Arnebrant, T. Lubricating properties of the initial salivary pellicle-An AFM study. Biofouling 2003, 19, 365-369.

126. Cárdenas, M.; Valle-Delgado, J.J.; Hamit, J.; Rutland, M.W.; Arnebrant, T. Interactions of hydroxyapatite surfaces: Conditioning films of human whole saliva. Langmuir 2008, 24, $7262-7268$.

127. Veeregowda, D.H.; Busscher, H.J.; Vissink, A.; Jager, D.-J.; Sharma, P.K.; van der Mei, H.C. Role of structure and glycosylation of adsorbed protein films in biolubrication. PLoS One 2012, 7, e42600.

128. Walz, A.; Stühler, K.; Wattenberg, A.; Hawranke, E.; Meyer, H.E.; Schmalz, G.; Blüggel, M.; Ruhl, S. Proteome analysis of glandular parotid and submandibular-sublingual saliva in comparison to whole human saliva by two-dimensional gel electrophoresis. Proteomics 2006, 6 , 1631-1639.

129. Veeregowda, D.H.; van der Mei, H.C.; de Vries, J.; Rutland, M.W.; Valle-Delgado, J.J.; Sharma, P.K.; Busscher, H.J. Boundary lubrication by brushed salivary conditioning films and their degree of glycosylation. Clin. Oral Investig. 2012, 16, 1499-1506.

130. Sotres, J.; Lindh, L.; Arnebrant, T. Friction force spectroscopy as a tool to study the strength and structure of salivary films. Langmuir 2011, 27, 13692-13700. 
131. Sotres, J.; Pettersson, T.; Lindh, L.; Arnebrant, T. Nanowear of salivary films vs. substratum wettability. J. Dent. Res. 2012, 91, 973-978.

132. Carlén, A.; Börjesson, A.C.; Nikdel, K.; Olsson, J. Composition of pellicles formed in vivo on tooth surfaces in different parts of the dentition, and in vitro on hydroxyapatite. Caries Res. 1998, $32,447-455$.

133. Dedinaite, A.; Lundin, M.; Macakova, L.; Auletta, T. Mucin-chitosan complexes at the solid-liquid interface: Multilayer formation and stability in surfactant solutions. Langmuir 2005, 21, 9502-9509.

134. Pettersson, T.; Dedinaite, A. Normal and friction forces between mucin and mucin-chitosan layers in absence and presence of SDS. J. Colloid Interface Sci. 2008, 324, 246-256.

135. Perez, E.; Proust, J.E. Forces between mica surfaces covered with adsorbed mucin across aqueous solution. J. Colloid Interface Sci. 1987, 118, 182-191.

136. Proust, J.E.; Baszkin, A.; Perez, E.; Boissonnade, M.M. Bovine submaxillary mucin (BSM) adsorption at solid/liquid interfaces and surface forces. Colloids Surf. 1984, 10, 43-52.

137. Nylander, T.; Arnebrant, T.; Glantz, P.O.; Baier, R.E. Interactions between layers of salivary acidic proline rich protein 1 (PRP1) adsorbed on mica surfaces. Prog. Colloid Polym. Sci. 1998, 108, 34-39.

138. Harvey, N.M.; Carpenter, G.H.; Proctor, G.B.; Klein, J. Normal and frictional interactions of purified human statherin adsorbed on molecularly-smooth solid substrata. Biofouling 2011, 27, 823-835.

139. Hahn Berg, I.C.; Lindh, L.; Arnebrant, T. Intraoral lubrication of PRP-1, statherin and mucin as studied by AFM. Biofouling 2004, 20, 65-70.

140. Sotres, J.; Madsen, J.B.; Arnebrant, T.; Lee, S. Adsorption and nanowear properties of bovine submaxillary mucin (BSM) films on solid surfaces: Influence of solution $\mathrm{pH}$ and substrate hydrophilicity. Langmuir 2013, submitted.

(C) 2013 by the authors; licensee MDPI, Basel, Switzerland. This article is an open access article distributed under the terms and conditions of the Creative Commons Attribution license (http://creativecommons.org/licenses/by/3.0/). 